\title{
Design of long-term stable concentrated colloidal dispersions in ionic liquids up to $473 \mathrm{~K}$
}

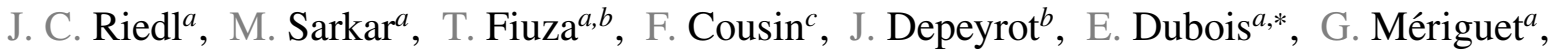 \\ R. Perzynski ${ }^{b}$ and V. Peyre ${ }^{b, *}$ \\ ${ }^{a}$ Sorbonne Université, CNRS, Laboratoire PHENIX, 4 place Jussieu, case 51, 75005, Paris, France. \\ ${ }^{b}$ Inst. de Fisica, Complex Fluid Group, Universidade de Brasília, Brasília, Brazil \\ ${ }^{c}$ Laboratoire Léon Brillouin, UMR 12 CNRS-CEA, CEA-Saclay, 91191, Gif-sur-Yvette, France.
}

\section{ARTICLE INFO}

\section{Keywords:}

Colloidal dispersions

Ionic liquids

Nanoparticles

Nanofluids

IOnanofluids

\begin{abstract}
A B S T R ACT
Hypothesis: One of the most promising field of application of ionic liquid-based colloids implies elevated temperatures. Therefore a careful design of the interface between the solid particles and the liquid solvent is crucial. A first optimization of the dispersions at room temperature should be followed by an analysis at high temperature and in the long run.

Experiments: The system chosen here consists of iron oxide nanoparticles (NPs) dispersed in ethylmethylimidazolium bistriflimide (EMIM TFSI). The key parameters of the solid/liquid interface, tuned at room temperature, are the surface charge density and the nature of the counterions. The thermal stability of these nanoparticle dispersions is then analysed on the short and long term up to $473 \mathrm{~K}$. A multiscale analysis is performed combining dynamic light scattering (DLS), small angle X-ray/neutron scattering (SAXS/SANS) and thermogravimetric analysis (TGA).

Findings: Following the proposed approach, ionic liquid-based colloidal dispersions of iron oxide NPs in EMIM TFSI stable in the long term can be obtained at least up to $473 \mathrm{~K}$ and NPs concentrations of 12 vol\% ( $\approx 30 \mathrm{wt} \%)$.
\end{abstract}

\section{Introduction}

Ionic liquids (ILs) have a variety of properties such as high electrochemical and thermal stabilities, low vapour pressures, their abilities to dissolve many chemical species, and modest conductivities making them interesting for several fields of applications. Some examples are found in synthesis and catalysis processes [52], seals and bearings[37], as well as energy related applications $[31,2]$ such as batteries, supercapacitors and thermoelectric applications $[6,27,11]$. However, ionic liquids also have some properties hindering their

Abbreviations: ILs, Ionic liquids; NPs: nanoparticles; MD: molecular dynamics; $\sigma$, solid's surface charge density; $\Theta_{i o n}^{\max }$, maximum charge density of a densely packed counterion monolayer; EMIMTFSI, ethylmethylimidazolium bistriflimide; DLS, Dynamic Light Scattering; SAXS, Small Angle X-Ray Scattering; SANS, Small Angle Neutron Scattering; TEM, Transmission electron microscopy, TGA, thermogravimetric analysis; $\mathrm{SBMIM}^{ \pm}$, deprotonated form of $\mathrm{HSBMIM}^{+}$; $\Phi_{\mathrm{NP}}$, nanoparticle's volume fraction; FAAS, flame atomic absorption spectroscopy; ESI-MS, electrospray ionization mass spectrometry; $\mathrm{S}(\mathrm{Q})$, structure factor; $\chi$, osmotic compressibility; HTfO, triflic acid; HTFSI, N,N-Bis(trifluoromethanesulfonyl)imide acid;HSBMIM TFSI, 1(4-Sulfobutyl)-3-methylimidazolium bistriflimide; HSBMIM TfO, 1-(4Sulfobutyl)-3-methylimidazolium triflate

${ }^{*}$ Corresponding authors

@ jesseriedl@web.de (J.C. Riedl); mitradeep.sarkar@gmail.com (M. Sarkar); thiagofiuza91@gmail.com (T. Fiuza); fabrice. cousin@cea. fr (F.

Cousin); jerome. depeyrot@gmail.com (J. Depeyrot);

emmanuelle. dubois@sorbonne-universite. fr (E. Dubois);

guillaume .meriguet@sorbonne-universite. fr (G. Mériguet);

regine. perzynski@sorbonne-universite. fr (R. Perzynski);

veronique. peyre@sorbonne-universite. fr (V. Peyre)

ORCID(s): $0000-0001-9361-5132$ (J.C. Riedl); 0000-0002-3431-0678 (M. Sarkar); $0000-0001-8859-0460$ (T. Fiuza); $0000-0001-7523-5160$ (F. Cousin); 0000-0002-1689-573x (J. Depeyrot); 0000-0001-8554-8486 (E. Dubois); 0000-0002-9921-0395 (G. Mériguet); 0000-0002-6634-6245 (R. Perzynski); $0000-0002-5129-1544$ (V. Peyre) industrial employment in some fields. For example they generally show elevated viscosities compared to molecular solvents which is connected to poor mass transport properties limiting the performance in energy applications at room temperature $[31,11]$. However, the viscosity reduces significantly when the ionic liquids are heated up whereby this disadvantage (almost) vanishes. Therefore, the use of ionic liquids at elevated temperatures is extremely interesting for applications. In addition, the performance of electrochemical devices is improved with increasing temperature. A way to further improve the system's properties at low and higher temperatures can be the addition of nanoparticles (NPs) to ionic liquids [19]. The addition of charged nanoparticles to electrolytes of electrochemical devices can, for instance, improve the thermoelectric properties compared to the solvent alone[46]. Such dispersions of NPs can be considered as potential heat transfer fluids thanks to their improved thermal properties [14].

Several approaches can stabilise nanoparticle dispersions in ionic liquids, ion layering of the ionic liquid around the nanoparticle being one possibility [54, 24, 42, 34, 32, 45]. It leads to repulsive (oscillatory) forces that can outrank the attractive Van der Waals forces between the nanoparticles and therefore colloidal stability is obtained. This was shown by molecular dynamics (MD) simulations [54] and X-ray pair distribution analysis[24] of nanoparticle dispersions in molten salts ${ }^{1}$ and some ionic liquids.

MD simulations of ionic liquids on flat surfaces show that this ion layering at the interface is controlled by the ratio $\kappa_{i o n}=\left|\sigma / \Theta_{i o n}^{\max }\right|$ with $\sigma$ being the solid's surface charge density and $\Theta_{i o n}^{\text {max }}$ being the maximum charge density of a densely

\footnotetext{
${ }^{1}$ analogue of ionic liquids with a melting temperature above $100^{\circ} \mathrm{C}$
} 
packed counterion ${ }^{2}$ monolayer [22]. For oxide NPs, $\sigma$ can be tuned in a limited range changing the acidity of the medium or the nature of the surface. On the other hand, the counterions can be changed in the system, either by the choice of the ionic liquid itself or by additional ions.

Several works analysing ionic-liquid based colloids evidenced that these additional ions influence the colloidal stability despite their low concentration compared to the ions from the ionic liquid. [34, 32, 17, 5, 40] Furthermore, nanostructure modifications are observed in interface studies between ionic liquids and chargeable surfaces like graphite[12] and gold[18] when adding extra small ions like $\mathrm{Li}^{+}$or $\mathrm{Cl}^{-}$. These ions (even at low concentrations) have an influence as they can locate at the interface.

The use of ionic liquids at elevated temperatures is extremely interesting for applications. To be suitable, the ionic liquidbased systems need to be long-term stable in these application conditions. Some ionic liquids are only decomposed at $400^{\circ} \mathrm{C}$ and higher temperatures when analysed in the short term. EMIM TFSI has one of the highest values and is therefore chosen as the ionic liquid here. [53]

In this work, first the colloidal stability of nanoparticle dispersions in the ionic liquid EMIM TFSI is analysed changing the surface charge density as well as the nature and concentration of counterions, choosing a system where they can be decoupled. The optimal surface charge density as well as the amount and nature of counterions is obtained for charged (magnetic) iron oxide based systems using optical microscopy, dynamic light scattering (DLS) and small angle X-ray scattering (SAXS) measurements. The two best compositions are then analysed by transmission electron microscopy (TEM). The second part of this work examines the long-term stability of the optimized colloids obtained at room temperature found in the first part. As the long-term temperature stability of ionic liquids can be up to a factor two lower than the short term one $[8,36]$, long-term analysis is necessary to determine suitability for applications. The colloidal dispersions are analysed up to $473 \mathrm{~K}\left(200^{\circ} \mathrm{C}\right)$ for hours combining dynamic light scattering (DLS), small angle X-ray/neutron scattering (SAXS/SANS) as well as thermogravimetric analysis (TGA) supported by DLS measurements over days.

\section{Materials and Methods}

\subsection{Preparation of ionic liquid-based colloids}

The products were purchased and used as received. They are listed in the ESI $\dagger(\mathrm{S} 1)$. The same batch of maghemite $\left(\gamma-\mathrm{Fe}_{2} \mathrm{O}_{3}\right)$ nanoparticles was used for all experiments. This batch was already used in reference [45], where the synthesis $[35,23]$ and the sample characteristics are described in detail. Briefly, sodium hydroxide solution was added to the initial acid ferrofluid stabilised with nitrate counterions till the point of zero charge was reached at $\mathrm{pH} \approx 7$. The free ions were washed off several times with ultra-pure water. After a size sorting process, the fraction kept has a lognormal size distribution with a median diameter $\mathrm{d}_{\mathrm{NP}}=8.7 \mathrm{~nm}$ with a poly-

\footnotetext{
${ }^{2}$ ions of opposite charge compared to the surface
}

dispersity index of $\sigma=0.3$. The particles can be charged by protonation of the oxide surface with four different strong acids to form hydroxyl groups: N,N-Bis(trifluoromethanesulfonyl)imide acid (HTFSI), triflic acid (HTfO), 1-(4-Sulfobutyl)-3-methylimidazolium bistriflimide (HSBMIM TFSI), and 1-(4-Sulfobutyl)-3-methylimidazolium triflate (HSBMIM TfO). The charge increases while decreasing the $\mathrm{pH}$ and it is compensated by the deprotonated form of these four acids, shown in Figure 1. Note that the acids HSBMIM TFSI and HSBMIM TfO are composed of two entities: the protonated cation $\mathrm{HSBMIM}^{+}$and the anion $\mathrm{TFSI}^{-}$or $\mathrm{TfO}^{-}{ }^{3}$. The deprotonated form is thus composed of the zwitterion SBMIM $^{ \pm}$ and the anion. In HSBMIM TFSI and HSBMIM TfO, the ratio of $\mathrm{SBMIM}^{ \pm}$and $\mathrm{TFSI}^{-}$or $\mathrm{TfO}^{-}$can be varied by adding first HTFSI or HTfO and then SBMIM $^{ \pm}$(which is a powder).

All the initial aqueous dispersions were mixed with a similar volume of the ionic liquid EMIM TFSI (see Figure 1) and the water was removed by freeze-drying. The resulting nanoparticle volume fraction $\Phi_{\mathrm{NP}}$ in the ionic liquid of around $1 \mathrm{vol} \%$ and the concentration of the initial counterions was hence kept.

More concentrated dispersions were obtained from the most stable samples by ultracentrifugation using an Optima 70 device and a type $100 \mathrm{Ti}$ fixed-angle rotor from the company Beckman Coulter, USA. The samples were centrifuged at 55 $000 \mathrm{rpm}(243000 \mathrm{~g})$ for 24 hours at $19{ }^{\circ} \mathrm{C}$. Separating the clear supernatant led to concentrated samples with nanoparticle volume fractions $\Phi_{\mathrm{NP}} \approx 10-20 \mathrm{vol} \%$ which were diluted with their supernatant to appropriate concentrations.

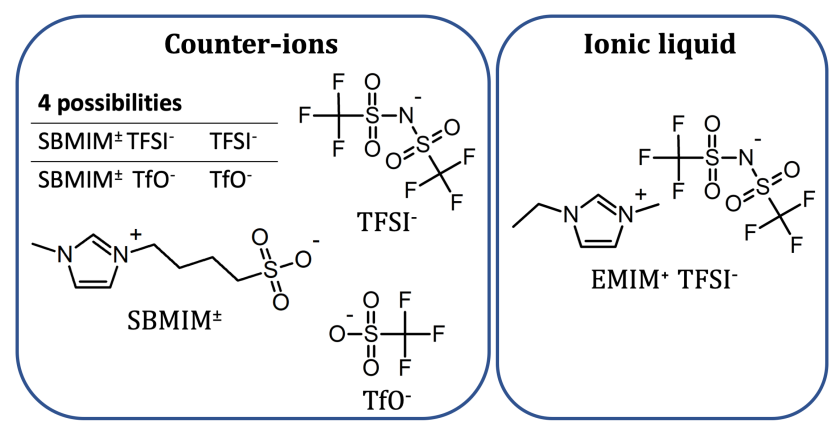

Figure 1: Structures of the ions 1-(4-butylsulfonate)3-methylimidazolium $\left(\mathrm{SBMIM}^{ \pm}\right)$, triflate $\left(\mathrm{TfO}^{-}\right)$, bis(trifluoromethylsulfonyl) amide $\left(\mathrm{TFSI}^{-}\right)$, and 1-ethyl3-methylimidazolium $\left(\mathrm{EMIM}^{+}\right)$.

\subsection{Analyses of the sample}

The ionic liquid-based colloids were analysed by optical microscopy in order to detect agglomerates on the micrometre scale. They were also analysed by transmission electron microscopy (TEM) using a JEOL-100 CX TEM in order to check that their transfer into the ionic liquid has not changed the shape of the nanoparticles compared to the initial sample in water, i.e there is no dissolution. A droplet of the ionic liquid-based ferrofluids (diluted to around 0.01vol\%

\footnotetext{
${ }^{3}$ Note that these two acids are ionic liquids when pure
} 
with pure ionic liquid) was deposited on a carbon-coated copper grid and the back-side of the grid was swiped over a paper to remove as much liquid as possible. Note that TEM can not give an insight on the dispersion state of nanoparticle in solution because the preparation of the sample for the TEM measurements can affect the sample (e.g. interparticle interactions) due to dilution, exposure to humidity of the air and small analysed volumes.

On the micron scale, if no major agglomeration is observed in optical microscopy, the samples were analysed by dynamic light scattering (DLS). Light scattering measurements were performed using Vasco, VascoFlex or VascoKin DLS Particle Analyzers from Cordouan Technologies to study translational diffusion properties of the ionic liquid-based colloids. Note that the viscosity of the ionic liquid-based colloids is difficult to know accurately as it depends on water traces [43] and on the other additives in the dispersions. However, performed together with other techniques, DLS can give complementary information and the properties of the samples can be analysed in the long-term. As measurements are performed at $1 \mathrm{vol} \%$ of NPs, only apparent hydrodynamic diameters $\mathrm{d}_{H}$ are given (see details in the ESI $\dagger$ section S2).

Light scattering was coupled with small angle neutron scattering (SANS) up to $473 \mathrm{~K}\left(200^{\circ} \mathrm{C}\right)$ for hours. The setup and the details of the experiments performed on the PAXY spectrometer at the LLB facility (CEA Saclay, France) are described in detail in the ESI $\dagger$ section S3. Three different configurations were used leading to an accessible Q-range of $0.005 \AA^{-1}$ to $0.2 \AA^{-1}$.

SAXS experiments were carried out with a laboratory instrument (XEUSS 2.0). The beam energy was fixed at $8 \mathrm{keV}$ $(\lambda=1.54 \AA)$. The sample to detector distance was $2.48 \mathrm{~m}$ to yield an accessible Q-range of $0.0045 \AA^{-1}-0.2 \AA^{-1}$. The samples absorb X-rays due to the ionic liquid EMIM TFSI and due to the iron atoms. Therefore thin capillaries were needed and the best compromise between absorption and scattered signal was obtained with $0.1 \mathrm{~mm}$ thick borosilicate capillaries from Vitrocom ${ }^{\circledR}$. However, the wall thickness of 0.07 $\mathrm{mm}$ has an error of $\pm 20 \%$ and the interior thickness error is $\pm 10 \%$. As a consequence, absolute intensity could hardly be determined and the high $\mathrm{Q}$ region $\left(>0.1 \AA^{-1}\right)$ was rather noisy. As a conclusion, the nanoparticle volume fraction together with the SANS measurements were used to adjust the SAXS curves to absolute intensities.

The SAXS and SANS form factor P(Q) of the nanoparticles were obtained from the extrapolation at $0 \mathrm{vol} \%$ of several dilute colloidal dispersions in water with low interparticle interactions. See the ESI of [45] for details and the corresponding curves. These form factors were used to determine structure factors $\mathrm{S}(\mathrm{Q})$ from the measured intensity $\mathrm{I}(\mathrm{Q})$ using:

$$
S(Q)=\frac{I(Q)}{\Delta \rho^{2} V_{\mathrm{NP}} \Phi_{\mathrm{NP}} P(Q)}
$$

where $\Delta \rho^{2}$ is the contrast and $V_{\mathrm{NP}}$ an average volume of the NP. The value of $S(Q \rightarrow 0)$ therefore compares the studied dispersions to a "reference" dispersion of individual nanopar- ticles without interparticle interaction. The difference can be due to interparticle interaction or to a change of the volume/form of the scattering objects (for example formation of small aggregates).

Isothermal and ramped temperature thermogravimetric analysis (TGA) were performed with a TGA 550 from TA Instruments. All samples (about $10-20 \mathrm{mg}$ with the mass precision $\pm 0.1 \mu \mathrm{g}$ ) were measured in platinum pans and some samples were also measured in an alumina crucible. The nitrogen gas flow was $40 \mathrm{~mL} / \mathrm{min}$. Short-term temperature stabilities were conducted with a heating rate of $10^{\circ} \mathrm{C} / \mathrm{min}$ from room temperature up to $600^{\circ} \mathrm{C}$. Long-term temperature stabilities were measured at isothermal mode for several hours at the specified isothermal temperature with a heating rate of $20^{\circ} \mathrm{C} / \mathrm{min}$ from room temperature up to this temperature.

The nanoparticle volume fractions were deduced from the iron concentration determined by flame atomic absorption spectroscopy (FAAS) taking a density of $4.87 \mathrm{~g} / \mathrm{cm}^{3}$ [48]. The technical details and the implementation of the techniques are detailed in the ESI of ref[45].

\section{Results and discussion}

\subsection{Decoupled analysis of the optimal counterion concentrations at $1 \mathrm{vol} \%$ of nanoparticles}

In a recent publication [45] it was demonstrated that colloidally long-term stable dispersions of oxide nanoparticles in EMIM TFSI can be prepared composed of positive nanoparticles with $\mathrm{SBMIM}^{ \pm} \mathrm{TFSI}^{-}$assumed to be at the solid liquid interface. Here, the role of both species (the zwitterion $\mathrm{SBMIM}^{ \pm}$and the anion $\mathrm{TFSI}^{-}$) is analysed in a decoupled way. In addition, $\mathrm{SBMIM}^{ \pm} \mathrm{TFSI}^{-}$is replaced by $\mathrm{SBMIM}^{ \pm} \mathrm{TfO}^{-}$in order to assess at the influence of the anion, $\mathrm{TfO}^{-}$being smaller than $\mathrm{TFSI}^{-}$, its charge coming from a different chemical group.

For this purpose, in a first step the surface charge density is changed in water thanks to $\mathrm{pH}$, beginning with dispersions at $1 \mathrm{vol} \%$ of NPs. Adding HTFSI or HTfO to the neutral nanoparticles at the point of zero charge, the particles become positive with $\mathrm{TFSI}^{-}$or $\mathrm{TfO}^{-}$counterions, the charge increasing while $\mathrm{pH}$ decreases. The concentration of HTFSI and HTfO was adapted to reach $\mathrm{pH}$ values between 5 and 1.3 which converts here to a surface charge between around 4 and $30 \mu \mathrm{C} / \mathrm{cm}^{2}$ [30] (the equivalent concentrations of the added acids are between 0.0035 and $0.075 \mathrm{~mol} / \mathrm{L}$ ). Note that lowering the $\mathrm{pH}$ too much can lead to the dissolution of the particles.

Adding the zwitterion $\mathrm{SBMIM}^{ \pm}$to these samples allows to introduce the second counterion independently so that the ratio of $\mathrm{SBMIM}^{ \pm}$to $\mathrm{TFSI}^{-}$or $\mathrm{TfO}^{-}$can be adjusted.

After the transfer to the ionic liquid keeping the NPs concentration at $1 \mathrm{vol} \%$, the samples are analysed by DLS measurements at room temperature and the deduced apparent hydrodynamic diameters $\mathrm{d}_{H}$ are depicted in Figure 2. Note that the process can be performed in one step by simply adding HSBMIM TFSI or HSBMIM TfO, leading to a ratio SBMIM $^{ \pm} /$ $\mathrm{TFSI}^{-}$or $\mathrm{TfO}^{-}$equals one. These cases are shown as a blue 
line in Figure 2.

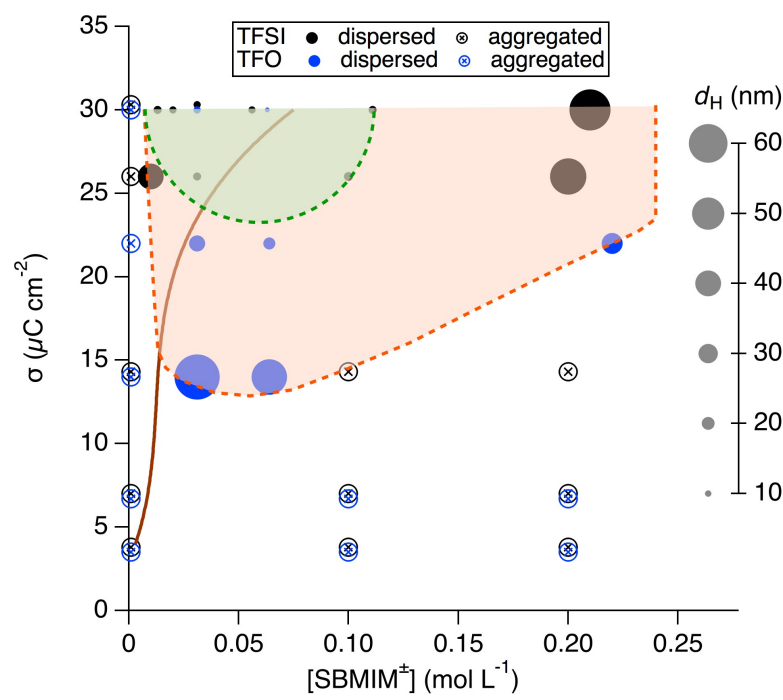

Figure 2: Map of the nanostructure of the dispersions of nanoparticles in EMIM TFSI from the DLS measurements as a function of the charge $\sigma$ of the NPs and of the added concentration of $\mathrm{SBMIM}^{ \pm}$. Each circle is a sample with either HTFSI (black disks) or HTFO (blue disks) added to introduce a charge on the NPs. The surface of the disk is related to the apparent size extracted from DLS as indicated on the scale on the right. The brown line corresponds to the amount of SBMIM $^{ \pm}$equal to the amount of HTFSI or HTFO added to obtain the indicated charge density $\sigma$. The green area indicates the region where the best samples are obtained. The red area indicates the region where samples are dispersed however with aggregates up to $60 \mathrm{~nm}$.

Several observations can be made with these experiments at a volume fraction $\Phi_{\mathrm{NP}} \approx 1 \%$.

1) Nanoparticles with $\mathrm{TFSI}^{-}$or $\mathrm{TfO}^{-}$counterions alone are flocculated systems whatever the surface charge densities analysed here (around 4 to $30 \mu \mathrm{C} / \mathrm{cm}^{2}$ ).

2) Adding $\mathrm{SBMIM}^{ \pm}$to these systems can stabilize the nanoparticles if the surface charge density (determined by the concentration of the $\mathrm{TFSI}^{-}$or $\mathrm{TfO}^{-}$ions added) is high enough which means that $\mathrm{SBMIM}^{ \pm}$localises at least partly at the solid liquid interface.

3) In these latter colloidally stable systems, a higher surface charge density leads to a lower apparent hydrodynamic diameter $\mathrm{d}_{H}$ for an $\mathrm{SBMIM}^{ \pm}$concentration in the range 0.01$0.1 \mathrm{~mol} / \mathrm{L}$ for both $\mathrm{TfO}^{-}$or $\mathrm{TFSI}^{-}$added ions.

4) In addition, the nature of the first ions introduced $\left(\mathrm{TfO}^{-}\right.$ or $\mathrm{TFSI}^{-}$) can influence the apparent hydrodynamic diameter $\mathrm{d}_{H}$ for the same counterion concentrations and surface charge density. For example for a surface charge density of $14 \mu \mathrm{C} / \mathrm{cm}^{2}\left(0.014 \mathrm{~mol} / \mathrm{L} \mathrm{TfO}^{-}\right.$or TFSI ${ }^{-}$introduced with the added acid) and a SBMIM ${ }^{ \pm}$concentration in the range 0.07 $0.1 \mathrm{~mol} / \mathrm{l}$ the apparent hydrodynamic diameter for nanoparticle dispersions containing $\mathrm{TfO}^{-}$is around $60 \mathrm{~nm}$, although it is bigger than $100 \mathrm{~nm}$ for dispersions with $\mathrm{TFSI}^{-}$.

5) At large enough surface charge density, the apparent hy- drodynamic diameter $\mathrm{d}_{H}$ is influenced by the added zwitterion $\mathrm{SBMIM}^{ \pm}$concentration. Starting from aggregated systems with only $\mathrm{TFSI}^{-}$or $\mathrm{TfO}^{-}$counterions, the addition of $\mathrm{SBMIM}^{ \pm}$first decreases the apparent hydrodynamic diameter $\mathrm{d}_{H}$, which increases again at higher $\mathrm{SBMIM}^{ \pm}$concentrations. The lowest apparent hydrodynamic diameter $\mathrm{d}_{H}$ are observed at the maximum surface charge density $\sigma \approx 30$ $\mu \mathrm{C} / \mathrm{cm}^{2}$ which corresponds to a concentration of $0.075 \mathrm{~mol} / \mathrm{L}$ of added acid. SAXS confirms that there is a change in the nanostructure while changing the concentration of SBMIM ${ }^{ \pm}$ for a given charge (see section S2 in ESI $\dagger$ ). This modifies the ratio of $\mathrm{SBMIM}^{ \pm}$to $\mathrm{TFSI}^{-}$or $\mathrm{TfO}^{-}$ions introduced. A 1:1 ratio leads to (one of) the lowest apparent hydrodynamic diameter $\mathrm{d}_{H}$ and can be added in one step in the form of HSBMIM TFSI or HSBMIM TfO. This makes the process easier and faster and it is thus used for further experiments as it is also more interesting for applications. SAXS also proves that several independent preparations with this composition are very reproducible (see section S2 in ESI $\dagger$ ).

\subsection{Room temperature colloidal stabilities}

Among the nanoparticle dispersions with the lowest apparent hydrodynamic diameter $\mathrm{d}_{H}$ analysed in section 3.1 the most interesting for applications (easiest to prepare) are analysed with transmission electron microscopy (TEM) and the images are depicted in Figure 3. They show for both nanoparticles with $\mathrm{SBMIM}^{ \pm} \mathrm{TFSI}^{-}$and $\mathrm{SBMIM}^{ \pm} \mathrm{TfO}^{-}$interfacial species that the shape of the nanoparticles is not changed compared to the initial sample in water (TEM picture given in ref[45]). Figure 3 shows that the nanoparti-

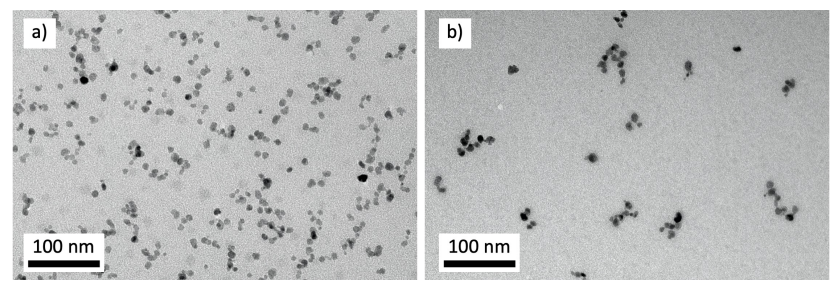

Figure 3: TEM images of nanoparticles dispersed in the ionic liquid EMIM TFSI with a) $\mathrm{SBMIM}^{ \pm} \mathrm{TFSI}^{-}$counterions and b) $\mathrm{SBMIM}^{ \pm} \mathrm{TfO}^{-}$interfacial species. The ion structures are depicted in Figure 1. Both samples were diluted to a nanoparticle volume fraction $\Phi_{\mathrm{NP}} \approx 0.01$ vol\% with pure EMIM TFSI before the deposition of the ionic liquid-based colloid on the TEM grid.

cles do not appear organized in the same way with SBMIM ${ }^{ \pm}$ $\mathrm{TfO}^{-}$and $\mathrm{SBMIM}^{ \pm} \mathrm{TFSI}^{-}$: they appear rather well dispersed with SBMIM $^{ \pm}$TFSI $^{-}$although small loose clusters are observed with $\mathrm{SBMIM}^{ \pm} \mathrm{TfO}^{-}$. Although the observation is direct (no drying), the dispersion is highly diluted and the measure itself may modify the system, which does not allow us drawing a definite conclusion.

Figure 4 shows SANS measurements for nanoparticles dispersed in EMIM TFSI with SBMIM ${ }^{ \pm}$TFSI $^{-}$and SBMIM $^{ \pm}$ $\mathrm{TfO}^{-}$interfacial species, plotted as structure factors $\mathrm{S}(\mathrm{Q})$. Interparticle interactions can be deduced from the value of the structure factor $\mathrm{S}(\mathrm{Q})$ extrapolated to $\mathrm{Q}=0 \AA^{-1}$. If 
$\mathrm{S}(\mathrm{Q} \rightarrow 0)$ is larger or smaller than 1 , the system is attractive or repulsive, respectively. The values for $\mathrm{S}(\mathrm{Q} \rightarrow 0)$ are given in the inset of Figure 4 as a function of the temperature and in Figure 5 as a function of the nanoparticle volume fraction $\Phi_{\mathrm{NP}}$. Figure 5 shows the values of $\mathrm{S}(\mathrm{Q} \rightarrow 0)$ determined from SANS and SAXS measurements for the same sample batch. From both SAXS and SANS it can be deduced that the global interparticle interactions of the system with SBMIM $^{ \pm}$ $\mathrm{TfO}^{-}$interfacial species is attractive $(\mathrm{S}(\mathrm{Q} \rightarrow 0)>1)$ and that of the system with $\mathrm{SBMIM}^{ \pm} \mathrm{TFSI}^{-}$interfacial species is repulsive $(\mathrm{S}(\mathrm{Q} \rightarrow 0)<1)$. This shows that not only the zwitterion $\mathrm{SBMIM}^{ \pm}$stabilises the nanoparticles but that introducing the additional counterions $\mathrm{TfO}^{-}$or $\mathrm{TFSI}^{-}$also plays a role in the nanostructure at the nanoparticle interface. This means that they need to be situated at the interface (at least in parts) to influence the nanoparticle interactions.

Apparent hydrodynamic diameter $\mathrm{d}_{H}$ deduced from DLS measurements at $\Phi_{\mathrm{NP}}=1 \%$ for the nanoparticles with SBMIM $^{ \pm}$TFSI $^{-}$interfacial species is approximately $12 \mathrm{~nm}$ (as for the initial water samples[45]), which proves that no aggregates are present. On the other hand, the sample with $\mathrm{SBMIM}^{ \pm} \mathrm{TfO}^{-}$interfacial species shows an average apparent hydrodynamic diameter $\mathrm{d}_{H} \approx 25 \mathrm{~nm}$, which suggests that small aggregates are present in the order of a few particles per aggregate.

Summarizing, the results obtained with small angle scattering, DLS and TEM at room temperature match well. There is a difference between the two samples as inferred from the TEM images (Figure 3). In the sample with SBMIM ${ }^{ \pm}$ TFSI $^{-}$interfacial species, the system's interparticle interactions are repulsive, which is consistent with the low apparent hydrodynamic diameter. The sample with $\mathrm{SBMIM}^{ \pm} \mathrm{TfO}^{-}$ interfacial species is also colloidally stable, however, the interparticle interaction is slightly attractive as deduced from SANS and SAXS measurements. This is consistent with the higher average apparent hydrodynamic diameter at $\Phi_{\mathrm{NP}}=1 \%$ $\mathrm{d}_{H} \approx 25 \mathrm{~nm}$ representing around a few particles per cluster. Besides their differences both samples are long-term stable at $\Phi_{\mathrm{NP}}=1 \%$ at least for one year as shown by SAXS measurements (Figure S2 of the ESI $\dagger$ ).

However, at larger concentration, attractive ionic liquidbased colloids can lead to a phase separation, as observed for citrated iron oxide NPs of similar size with various counterions in another IL. [33] Hence, here only the nanoparticle system with $\mathrm{SBMIM}^{ \pm} \mathrm{TFSI}^{-}$interfacial species is concentrated as it is repulsive. The interparticle interactions are analysed by SAXS and SANS measurements as a function of the nanoparticle volume fraction $\Phi_{\mathrm{NP}}$. The deduced values of $\mathrm{S}(\mathrm{Q} \rightarrow 0)$ are shown in Figure 5. For a repulsive system, $\mathrm{S}(\mathrm{Q} \rightarrow 0)$ equals the osmotic compressibility $\chi$. For individually dispersed particles, $\chi$ is related to the osmotic pressure $\Pi$.

$$
S(Q \rightarrow 0)=\chi\left(\Phi_{\mathrm{NP}}\right)=\frac{k T}{V_{N P}} \frac{\partial \Phi_{\mathrm{NP}}}{\partial \Pi}
$$

with $\mathrm{V}_{\mathrm{NP}}$ the average volume of a nanoparticle. At a low volume fraction $\Phi_{\mathrm{NP}}$, the second order virial development of $\Pi$

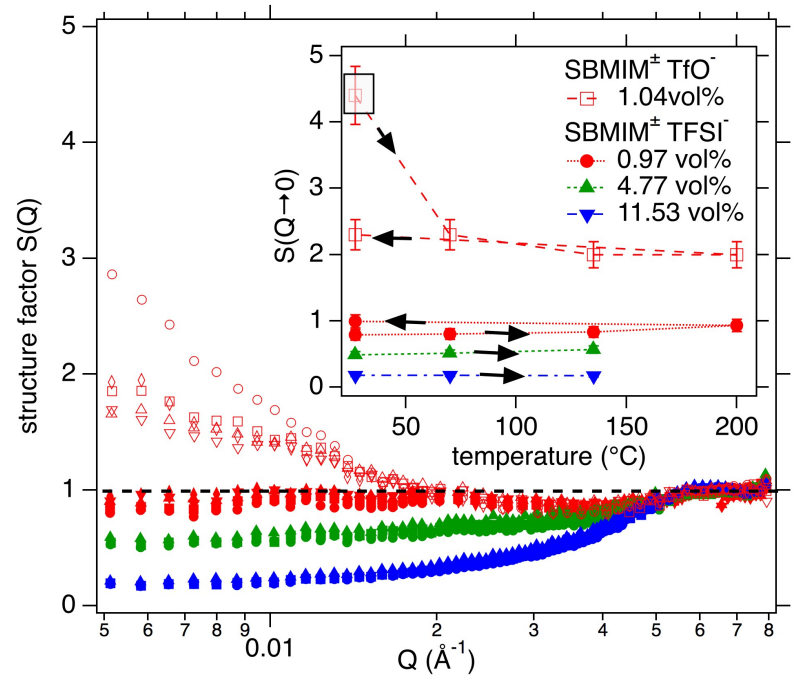

Figure 4: Structure factors $S(Q)$ at different temperatures $\left(27^{\circ} \mathrm{C}(\circ), 70^{\circ} \mathrm{C}(\square), 135^{\circ} \mathrm{C}(\triangle)\right.$, and $473 \mathrm{~K}\left(200^{\circ} \mathrm{C}\right)(\nabla)$ and back at $\left.27^{\circ} \mathrm{C}(\diamond)\right)$ obtained by small angle neutron scattering (SANS) as a function of the scattering vector $Q$ for nanoparticle dispersions in EMIM TFSI. One sample with $\mathrm{SBMIM}^{ \pm} \mathrm{TfO}^{-}$ interfacial species at $\Phi_{\mathrm{NP}}=1.04$ vol\% (empty red symbols) and three samples with $\mathrm{SBMIM}^{ \pm} \mathrm{TFSI}^{-}$interfacial species at $\Phi_{\mathrm{NP}}=0.97,4.77$ or 11.53 vol\% (full red, green, and blue symbols, respectively) are shown. The ion structures are depicted in Figure 1. The data in the grey box is an artefact (see text for details). The inset gives the values for $S(Q \rightarrow 0)$ as a function of temperature. The lines are guides to the eye. The arrows indicate the heating cycle. Note that both concentrated sample cells leaked at $200^{\circ} \mathrm{C}$. Therefore, no further SANS measurements could be obtained (SAXS and DLS could be performed with the remaining sample, the latter being plotted in the inset of Figure 5)

relates $\chi\left(\Phi_{\mathrm{NP}}\right)$ with the second order virial coefficient $\mathrm{A}_{2}$ by: $\chi\left(\Phi_{\mathrm{NP}}\right)=\left(1+2 A_{2} \Phi_{\mathrm{NP}}\right)^{-1}$. The osmotic compressibility $\chi\left(\Phi_{\mathrm{NP}}\right)$ decreases with increasing nanoparticle volume fraction $\Phi_{\mathrm{NP}}$. Therefore the interparticle interaction in the dispersion is repulsive as the two-bodies interaction term $\mathrm{A}_{2}$ is positive. In this case, the Carnahan-Starling expression for effective hard spheres $[9,4]$ can be used to analyse the compressibility $\chi$ up to large volume fractions as in references $[5,47,26,51,13,28,7]$. In this model an effective volume fraction $\Phi_{\text {eff } f}$ accounts for the decrease of the interparticle interaction through a characteristic length $\kappa^{-1}$ which is the analogue of the Debye screening length of interparticle electrostatic repulsion in standard polar solvents. Equation (3) describes the compressibility

$$
\chi\left(\Phi_{\mathrm{NP}}\right)=\chi_{C S}\left(\Phi_{e f f}\right)=\frac{\left(1-\Phi_{e f f}\right)^{4}}{1+4 \Phi_{e f f}+4 \Phi_{e f f}^{2}-4 \Phi_{e f f}^{3}+\Phi_{e f f}^{4}}
$$

with 


$$
\begin{aligned}
\Phi_{e f f}=\Phi_{\mathrm{NP}} \frac{d_{e f f}^{3}}{d_{N P}^{3}}= & \Phi_{\mathrm{NP}}\left(1+\frac{2 \kappa^{-1}}{d_{N P}}\right)^{3} \\
& \text { and } \quad d_{e f f}=d_{N P}+2 \kappa^{-1}
\end{aligned}
$$

and the two-bodies interparticle interaction $\mathrm{A}_{2}$ is then

$$
A_{2}=4 \frac{\Phi_{e f f}}{\Phi_{\mathrm{NP}}} .
$$

The experimental data of the compressibility are fitted in Figure 5 with the Equations (3) and (4). The best fit is obtained for a fitting parameter $\kappa^{-1} \approx 1.0 \mathrm{~nm}$ taking the average diameter $\mathrm{d}_{N P}=8.7 \mathrm{~nm}$. The extension of the interfacial area modified by the solid surface of the NP is therefore 3-5 $\kappa^{-1}$, hence $3-5 \mathrm{~nm}$. The two-bodies interparticle interaction $\mathrm{A}_{2}$ is then $\mathrm{A}_{2}=7.8 \pm 0.5$ which is almost the double of the hard sphere value $\mathrm{A}_{2}^{H S}=4[9]$ and of the values obtained for citrate coated oxide nanoparticles in ethylammonium nitrate with sodium counterions and smaller NPs $\left(\mathrm{A}_{2}=4.6\right)$. $[32,5]$

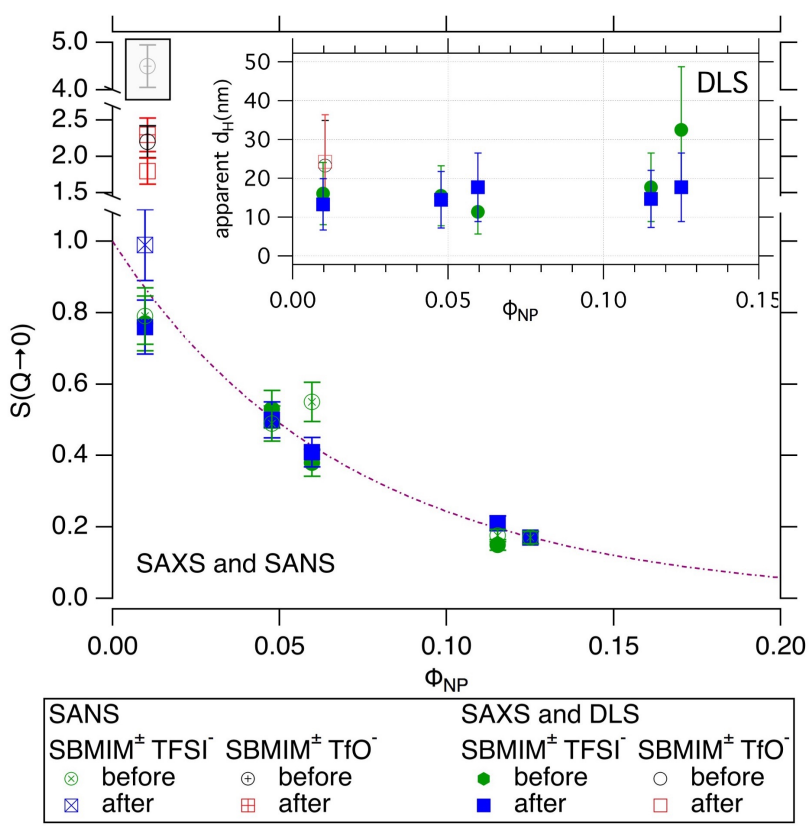

Figure 5: $S(Q \rightarrow 0)$ deduced from $S A X S$ and SANS measurements at room temperature for $\mathrm{SBMIM}^{ \pm} \mathrm{TFSI}^{-}$interfacial species before and after heating the sample for several hours up to $473 \mathrm{~K}\left(200^{\circ} \mathrm{C}\right)$ are depicted as a function of the nanoparticle volume fraction $\Phi_{\mathrm{NP}}$. The data in the grey box is an artefact (see text for details). The dashed-dotted line corresponds to the fit of the data for the repulsive samples with Equations (3) and (4) using $\phi_{\text {eff }} / \Phi_{\mathrm{NP}}=1.82$ (two-bodies interparticle interaction quantified by the second virial coefficient $A_{2}=7.8 \pm 0.5$ ). The inset shows apparent hydrodynamic diameter $\mathrm{d}_{H}$ at room temperature before and after heating the samples for several hours up to $200^{\circ} \mathrm{C}$ as a function of the nanoparticle volume fraction $\Phi_{\mathrm{NP}}$ for both $\mathrm{SBMIM}^{ \pm} \mathrm{TFSI}^{-}$and $\mathrm{SBMIM}^{ \pm} \mathrm{TfO}^{-}$interfacial species. The ion structures are depicted in Figure 1.

\subsection{Thermal stabilities}

The long-term stability of the samples needs to be guaranteed on a large range of temperatures to be suitable for applications. Therefore, the two optimized systems at $\sigma=30$ $\mu \mathrm{C} / \mathrm{cm}^{2}$ already studied in the previous sections are analysed at high temperatures. These systems are nanoparticles with $\mathrm{SBMIM}^{ \pm} \mathrm{TfO}^{-}$interfacial species $\left(\Phi_{\mathrm{NP}}=1.04 \mathrm{vol} \%\right)$ and nanoparticles with $\mathrm{SBMIM}^{ \pm} \mathrm{TFSI}^{-}$interfacial species $\left(\Phi_{\mathrm{NP}}=0.97 \mathrm{vol} \%, 4.77 \mathrm{vol} \%\right.$, and $\left.11.53 \mathrm{vol} \%\right)$. Several techniques are coupled on different length scales.

Let us begin with the in-situ measurements of SANS and DLS up to $473 \mathrm{~K}\left(200^{\circ} \mathrm{C}\right)$. Given the limited SANS time, few temperatures were chosen $\left(27^{\circ} \mathrm{C}, 70^{\circ} \mathrm{C}, 135^{\circ} \mathrm{C}, 200^{\circ} \mathrm{C}\right.$, and back at $27^{\circ} \mathrm{C}$ ). For each step, DLS was performed after heating and enables checking that equilibrium is reached before measuring SANS. This is possible as the viscosity varies with temperature which influences the correlation function. Hence, stability of the correlation function means that viscosity and temperature are stable. DLS performed after SANS tells us whether some change occurred. One $\mathrm{T}$ step lasts around two hours, the whole cycle from $27^{\circ} \mathrm{C}$ back to $27^{\circ} \mathrm{C}$ around 10 hours. These DLS data are presented in ESI $\dagger$, section S3, Figure S4.

The structure factors are plotted in Figure 4 and the DLS is presented as an apparent $d_{H}$ in the inset of Figure 5 at $27^{\circ} \mathrm{C}$ before and after the heating cycle up to $200^{\circ} \mathrm{C}$. Note that SAXS before and after heating has also been performed (Figure 5) to complement the analysis.

Looking first at the two samples at $\Phi_{\mathrm{NP}} \approx 1$ vol\% with different interfacial species, they show a weak evolution after they stay at high temperature (Figure 4 and its inset showing $\mathrm{S}(\mathrm{Q} \rightarrow 0)$ ). Indeed, the strong initial decrease of intensity for nanoparticles with $\mathrm{SBMIM}^{ \pm} \mathrm{TfO}^{-}$interfacial species from $27^{\circ} \mathrm{C}$ to $70^{\circ} \mathrm{C}$ appears as an experimental artefact. The corresponding SAXS measurement at room temperature gives $\mathrm{S}(\mathrm{Q} \rightarrow 0)=2.2 \pm 0.2$, which matches with the SANS after the cycle. Moreover, the DLS is similar before and after heating. This artefact can be due to some pollution (water, solvent,...) that is removed reversibly while heating. Leaving aside this point, the values of $S(Q \rightarrow 0)$ show that for these nanoparticles with $\mathrm{SBMIM}^{ \pm} \mathrm{TfO}^{-}$interfacial species, the same small aggregates/weak attractions remain whatever the temperature. Both the repulsive and the attractive sample appear stable on the time scale of the experiment.

For the nanoparticles with $\mathrm{SBMIM}^{ \pm} \mathrm{TFSI}^{-}$interfacial species, the interaction remains repulsive whatever the temperature. However, due to a small increase of $\mathrm{S}(\mathrm{Q} \rightarrow 0)$ at $\Phi_{\mathrm{NP}}=$ $0.97 \%$ from $0.83 \pm 0.08$ to $0.98 \pm 0.10$ after heating, a longterm experiment was performed. The sample was heated in a glass tube for several days at three temperatures up to $170^{\circ} \mathrm{C}$ under vacuum and its state was checked with a DLS measurement at room temperature after each plateau. The results are summarised in Figure 6. The apparent hydrodynamic diameter is $\mathrm{d}_{H}=12 \pm 2 \mathrm{~nm}$ showing that the sample is long-term stable at high temperature, here during 18 days.

Analysing now by SANS, SAXS and DLS the behaviour of the concentrated samples based on nanoparticles with 


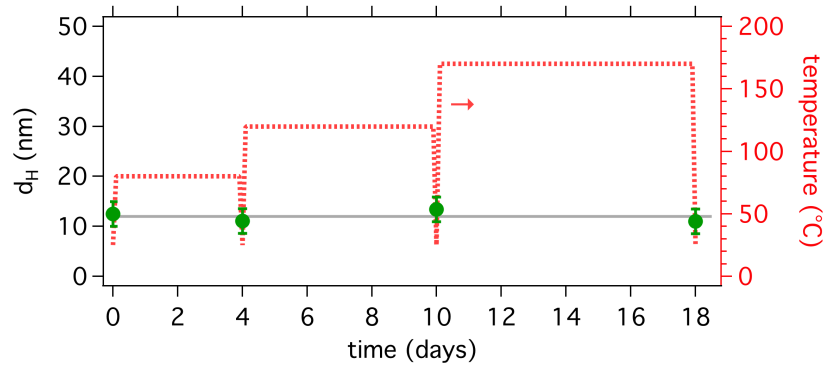

Figure 6: DLS measurements at room temperature for the iron oxide nanoparticle dispersion with $\mathrm{SBMIM}^{ \pm} \mathrm{TFSI}^{-}$interfacial species at a nanoparticle volume fraction $\Phi_{\mathrm{NP}}=0.97 \mathrm{vol} \%$ shown in Figure 4 and 5 . The sample was previously heated in a low vacuum of $\approx 10$ mbar in an open bottle at the indicated temperatures for days. Note that the maximum temperature of the heating device is $170^{\circ} \mathrm{C}$. These DLS measurements at room temperature give extra information about temperature cycling.

SBMIM $^{ \pm}$TFSI $^{-}$interfacial species, the results are quite analogous. Note however that both concentrated sample cells leaked at $200^{\circ} \mathrm{C}$, therefore no SANS could be performed at $200^{\circ} \mathrm{C}$ and back at $25^{\circ} \mathrm{C}$ after heating (see section $\mathrm{S} 3$ in ESI $\dagger$ ). SAXS and DLS after heating were nevertheless measured with the remaining sample.

At high temperature, the structure factors are close to those at room temperature and the compressibilities $S(Q \rightarrow 0)$, Figure 5) after heating are also quite similar (Figure 4). The apparent $\mathrm{d}_{H}$ differ before and after heating for the concentrated samples (Figure 5, inset), some increasing others decreasing. This is probably due to the error bar on these values due to the delicate measurement in the furnace, especially for concentrated samples.

All these elements converge towards a good stability of these concentrated samples at high temperature, keeping the same nanostructure and interaction.

These analysis have been complemented by TGA, which brings an indirect and global information while heating. It elucidates the temperatures that can be used for applications in the short and long term. The weight losses of ramped TGA measurements up to $600^{\circ} \mathrm{C}$ are depicted in Figure 7 for ionic liquids without and with nanoparticles (see the derivative weights in ESI). Several values can be given for the thermal degradation, with $\mathrm{T}_{\text {onset }}$ and $\mathrm{T}_{\text {start }}$ being among the most popular. $[36,15] \mathrm{T}_{\text {onset }}$ is defined by the intersection of the linear extrapolation of the weight loss at decomposition to the baseline weight, either from the beginning of the experiment or after a drying step. [15] This value gives higher decomposition temperatures than $\mathrm{T}_{\text {start }}$ that is the temperature at which the sample starts to loose weight. It is here defined for a derivative mass loss of $10 \% / 1 \mathrm{~h}$ for a heating rate of $10^{\circ} \mathrm{C} / \mathrm{min}$ (thus $1.67 \times 10^{-2} \% /{ }^{\circ} \mathrm{C}$, see Figure S5 in ESI $\dagger$ ). We will focus here on $\mathrm{T}_{\text {start }}$ values as this gives closer values to long-term applications.

Table 1 gathers the thresholds extracted from TGA either from our measurements (Figure 7 and Figure S5 in ESI $\dagger$, section S3.2.) or from literature when available. For the fer- rofluids with a nanoparticle volume fraction $\Phi_{\mathrm{NP}} \approx 1 \mathrm{vol} \%$. $\mathrm{T}_{\text {start }}=303 \pm 10^{\circ} \mathrm{C}$ for both $\mathrm{SBMIM}^{ \pm} \mathrm{TFSI}^{-}$and $\mathrm{SBMIM}^{ \pm}$ $\mathrm{TfO}^{-}$interfacial species although $\mathrm{T}_{\text {onset }}$ for $\mathrm{SBMIM}^{ \pm} \mathrm{TFSI}^{-}$ is around $20^{\circ} \mathrm{C}$ smaller than for the sample with $\mathrm{SBMIM}^{ \pm}$ $\mathrm{TfO}^{-}$interfacial species.

These curves can be compared to the data for pure EMIM TFSI. The literature values give $\mathrm{T}_{\text {onset }}$ in the range of 410 and $450^{\circ} \mathrm{C}$ and $\mathrm{T}_{\text {start }} \approx 320^{\circ} \mathrm{C},[8,3,39]$ values higher than for the ferrofluids analysed here. However, the ferrofluids may contain some water around the nanoparticles which would correspond to $0.15-0.3 \mathrm{vol} \%$ if one or two water layers were present at the interface. EMIM TFSI left at air thus containing $0.5 \mathrm{wt} \%$ has been measured. It gives $\mathrm{T}_{\text {onset }}=386 \pm 10^{\circ} \mathrm{C}$ and $\mathrm{T}_{\text {start }}=322 \pm 10^{\circ} \mathrm{C}$, lower than dry EMIM TFSI, however, higher than for the ferrofluids. The small difference between the nanoparticle dispersions could also be explained by the $\mathrm{TfO}^{-}$ions in the case of $\mathrm{SBMIM}^{ \pm} \mathrm{TfO}^{-}$interfacial species. EMIM TfO is indeed less stable than EMIM TFSI according both to literature $\left(\mathrm{T}_{\text {onset }}=348^{\circ} \mathrm{C}[20]\right)$ and to our measurements with absorbed water $(\approx 1.5 \mathrm{wt} \%)$.

Another element is however present in the ferrofluids: a mixture of HSBMIM and SBMIM $^{ \pm}$with an amount of around $2.5 \mathrm{wt} \%$ for $\Phi_{\mathrm{NP}} \approx 1 \mathrm{vol} \%$. Hence both pure HSBMIM TFSI and $\mathrm{SBMIM}^{ \pm}$have been measured, and appear less stable than EMIM TFSI. Note that the measurement of pure HSBMIM TFSI has to be done in an alumina crucible as it seems to decompose close to room temperature in a Pt pan (see Figure S6 ESI $\dagger$ ). Dry EMIM TFSI with $2.5 \mathrm{wt} \%$ of HSBMIM TFSI is also analysed and gives $\mathrm{T}_{\text {onset }}=401^{\circ} \mathrm{C}$ and $\mathrm{T}_{\text {start }}=295 \pm 10^{\circ} \mathrm{C}$, a little smaller than pure dry EMIM TFSI. The sample with an elevated nanoparticle volume fraction $\left(\Phi_{\mathrm{NP}}=4.77 \mathrm{vol} \%\right)$ gives $\mathrm{T}_{\text {start }}=283 \pm 10^{\circ} \mathrm{C}, 20^{\circ} \mathrm{C}$ lower than for both nanoparticle dispersions at around $1 \mathrm{vol} \%$ nanoparticles. The less stable component thus appears to be HSBMIM, much less stable than SBMIM $^{ \pm}$(Figure 7), however this should be taken with caution in the mixtures due to the possible influence of the nature of the pan and of the interaction between the components in the mixtures.

Note that partial evaporation of the sample also leads to a weight loss although the sample is not decomposed. EMIM TFSI for example is considered relatively volatile compared to other ionic liquids and its partial pressure at $200^{\circ} \mathrm{C}$ is 0.02 $\mathrm{Pa}$ (calculated with the values of ref[20]). This will be discussed in detail in the following section.

In addition, long term thermal stabilities are analysed in isothermal mode during 15h (see Figure S7 of the ESI $\dagger$ ). The first analysis in classical Pt crucibles shows that the weight loss for the ionic liquid EMIM TfO is smaller than $0.1 \%$ after 15 hours at $200^{\circ} \mathrm{C}$. For EMIM TFSI, the weight loss is a bit higher $(0.2 \%)$ and increases with the NPs volume fraction up to $1.5 \%$. The latter measurements reproduced in alumina crucible where the interference between HSBMIM and platinum is eliminated and the evaporation limited show a weight variation lower than $0.2 \%$ after 15 hours for both the solvent and the dispersions, pointing towards a good stability of the obtained dispersions. 
Table 1

Critical temperatures $\left(T_{\text {start }}\right.$ and $T_{\text {onset }}$ ) in Celsius degrees deduced from ramped thermogravimetric analysis (TGA) measurements. $\mathrm{T}_{\text {start }}$ gives the temperature at which the sample starts to loose weight, here defined for a derivative weight loss of $1.67 \times 10^{-2} \% /{ }^{\circ} \mathrm{C}$. $\mathrm{T}_{\text {onset }}$ is defined by the linear extrapolation of the weight loss at decomposition to the baseline at zero weight loss. All temperatures are given in ${ }^{\circ} \mathrm{C}$ and the error is $\pm 10^{\circ} \mathrm{C}$. losses due to evaporation are removed; ${ }^{i}$ for ionic liquid containing water.

\begin{tabular}{|c|c|c|c|c|c|c|c|}
\hline & \multicolumn{3}{|c|}{ measured } & \multicolumn{4}{|c|}{ literature } \\
\hline & $\mathrm{T}_{\text {start }}$ & $\mathrm{T}_{\text {onset }}$ & $\begin{array}{l}\text { weight loss after } \\
10 \mathrm{~h} \text { at } 200^{\circ} \mathrm{C}\end{array}$ & $\mathrm{T}_{\text {start }}$ & $\mathrm{T}_{\text {onset }}$ & $\begin{array}{l}\mathrm{T}_{0.01 / 10 h} \\
\text { or } \mathrm{T}_{0.02 / 10 h}\end{array}$ & $\operatorname{Tmax}_{0.01 / \text { year }}$ \\
\hline $\begin{array}{l}\Phi_{\mathrm{NP}}=1.04 \mathrm{vol} \% \\
\text { SBMIM }^{ \pm} \text {TfO }^{-}\end{array}$ & 303 & 393 & & - & - & & \\
\hline $\begin{array}{l}\Phi_{\mathrm{NP}}=0.97 \mathrm{vol}^{2} \\
\mathrm{SBMIM}^{ \pm} \mathrm{TFSI}^{-}\end{array}$ & 303 & 374 & & - & - & & \\
\hline $\begin{array}{l}\Phi_{\mathrm{NP}}=4.77 \mathrm{vol}^{2} \\
\text { SBMIM }^{ \pm} \text {TFSI }^{-}\end{array}$ & 283 & 377 & & - & - & & \\
\hline EMIM TfO & $333^{i}$ & $373^{i}$ & $<0.1 \%$ & & $348[20]$ & & $185[20] *$ \\
\hline EMIM TFSI & $322^{i}$ & $386^{i}$ & $<0.1 \%[50]$ & $320[8]$ & $410-450[8,3,39]$ & $222[8]$ or $252[50]$ & $286[20] *$ \\
\hline $\begin{array}{l}\text { EMIM TFSI } \\
+2.5 \text { wt } \% \\
\text { HSBMIM TFSI }\end{array}$ & 295 & 401 & & - & - & & \\
\hline SBMIM $^{ \pm}$ & 274 & 325 & & & & & \\
\hline HSBMIM TFSI & 158 & 305 & & & & & \\
\hline $\mathrm{HSBMIM} \mathrm{HSO}_{4}$ & & & & & $327[16]$ & & \\
\hline HSPMIM TfO & & & & & $288[1]$ & & \\
\hline
\end{tabular}

\subsection{Discussion}

The colloidal stability of the analysed iron oxide nanoparticle dispersions is first discussed in general and then in regard of thermal stability.

A structuring of ions at the solid-liquid interface is proven to be the reason for colloidal stability in ionic liquids and molten salts. [54, 24] Simulations of ionic liquids on flat surfaces show that this ion layering at the interface is controlled by the ratio $\kappa_{i o n}=\left|\sigma / \Theta_{i o n}^{\max }\right|$ with $\sigma$ being the solid's surface charge density and $\Theta_{i o n}^{\max }$ being the maximum charge density of a densely packed counterion monolayer.[22] A multilayered structure of positive and negative ions is formed close to the solid surface if the ratio $\kappa_{i o n}$ is close to 0.5.[22, 21] Although this has been studied for one type of counterions only and although a curvature of the nanoparticle surface and surface roughness influence the situation, it is helpful for a discussion.

Experimentally, both $\sigma$ and $\Theta_{i o n}^{\max }$ are varied in the studied systems, $\Theta_{i o n}^{\max }$ through the nature, thus the size, of the ions and $\sigma$ through the acidity of the surrounding medium. Assuming a similar behaviour for the nanoparticle charge for the different counterions [30], $\sigma$ is here tuned between 4 and $30 \mu \mathrm{C} / \mathrm{cm}^{2}$.

The $\Theta_{i o n}^{\max }$ values of the analysed ions are $\left|\Theta_{E M I M^{+}}^{\max }\right| \approx 45$ $\mu \mathrm{C} / \mathrm{cm}^{2},[21]\left|\Theta_{T F S I^{-}}^{\max }\right| \approx 50 \mu \mathrm{C} / \mathrm{cm}^{2},[25]\left|\Theta_{\text {TfO }^{-}}^{\max }\right| \approx 100$ $\mu \mathrm{C} / \mathrm{cm}^{2}$ assuming that it is around half the size of the TFSI ${ }^{-}$ anion, and $\left|\Theta_{S B M I M^{ \pm}}^{\max }\right| \approx 35 \mu \mathrm{C} / \mathrm{cm}^{2}$ considering that SBMIM $^{ \pm}$is a BMIM $^{+}$cation with an extra sulfonate group leading to a slightly smaller $\Theta_{i o n}^{\max }$ value.

What can we learn from the results described above? Figure 8 shows two imaginable schemes of the nanoparticle (NP) ionic liquid (IL) interface for the case of $\mathrm{SBMIM}^{ \pm} \mathrm{TFSI}^{-}$ and $\mathrm{SBMIM}^{ \pm} \mathrm{TfO}^{-}$interfacial species at the maximal surface charge density.

a) The zwitterions $\mathrm{SBMIM}^{ \pm}$must locate at the solid-liquid interface as colloidal stability can only be achieved in their presence.

b) For the maximal surface charge density $\sigma$ (here $\approx 30 \mu \mathrm{C} /$ $\left.\mathrm{cm}^{2}\right)$, the ratio $\kappa_{i o n}=\left|\sigma / \Theta_{i o n}^{\max }\right|$ for the zwitterion $\mathrm{SBMIM}^{ \pm}$ is close to 1 meaning that a structure close to a monolayer would be formed on the nanoparticle surface if a sufficient amount of $\mathrm{SBMIM}^{ \pm}$is introduced. As $\mathrm{SBMIM}^{ \pm}$is a zwitterion this would not change the sign and value of the positive nanoparticle surface charge. The zwitterion SBMIM $^{ \pm}$ could hence increase the distance of an ion layering to the nanoparticle surface leading to a bigger repulsion between the nanoparticles.

c) With the negative sulfonate group close to the positive nanoparticle surface, the imidazolium end shows away from the nanoparticle surface, providing a positive charge on the top of the $\mathrm{SBMIM}^{ \pm}$layer, which can induce organisation. For this maximal surface charge, stable dispersions are obtained on a large range of $\mathrm{SBMIM}^{ \pm}$concentrations $(0.01$ $0.12 \mathrm{~mol} / \mathrm{L}$ ). Note that $0.01 \mathrm{~mol} / \mathrm{L}$ corresponds to around half a monolayer of $\mathrm{SBMIM}^{ \pm}$assuming all these zwitterions located at the nanoparticle interface, and that several hours of stirring are necessary to reach dispersions. This time decreases to a few seconds for higher concentration. It means that a mixed layer of $\mathrm{SBMIM}^{ \pm}$and $\mathrm{TFSI}^{-}$or $\mathrm{TFO}^{-}$species enables colloidal stabilization. The negative charge of the next layer can be the sulfonate of the $\mathrm{SBMIM}^{ \pm}$or $\mathrm{TfO}^{-}$or $\mathrm{TFSI}^{-}$depending on the composition. As the properties of 


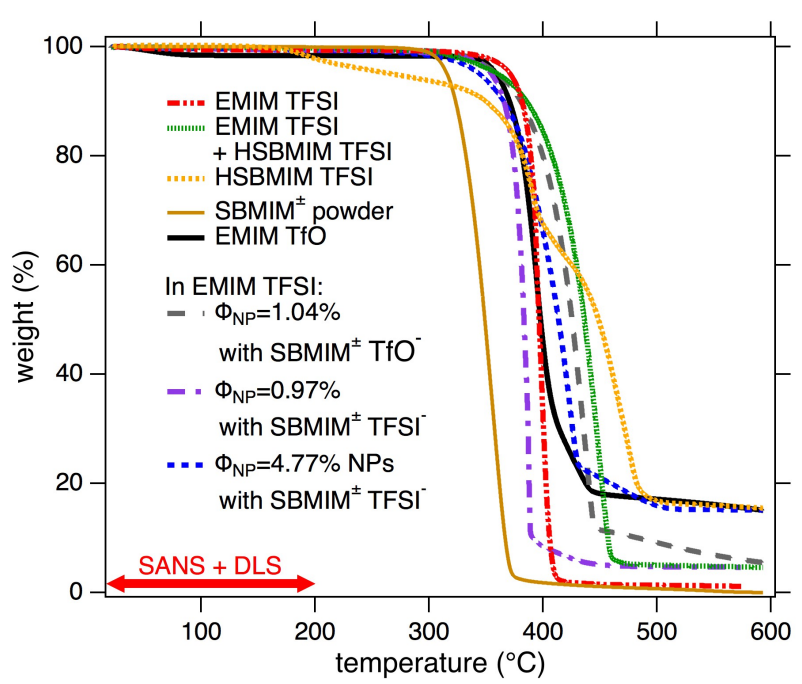

Figure 7: Ramped thermogravimetric analysis (TGA) measurements for the components of the nanoparticle dispersions in EMIM TFSI and for the resulting dispersions in EMIM TFSI. Pure EMIM TFSI and EMIM TfO are wet to mimic the worst case possible in the dispersions. Dry $\mathrm{SBMIM}^{ \pm}$and dry EMIM TFSI containing 2.5wt\% HSBMIM TFSI are also depicted, 2.5wt \% HSBMIM TFSI being the amount added while preparing the nanoparticle dispersions at 1 vol\% nanoparticles. The final dispersions with $\Phi_{\mathrm{NP}}=1.04$ vol $\%$ with $\mathrm{SBMIM}^{ \pm}$ $\mathrm{TfO}^{-}$interfacial species as well as $\Phi_{\mathrm{NP}}=0.97 \mathrm{vol} \%$ and a concentrated sample with $\Phi_{\mathrm{NP}}=4.77$ vol\% nanoparticles with $\mathrm{SBMIM}^{ \pm} \mathrm{TFSI}^{-}$are also plotted. Note that all measurements are performed in Pt pans except HSBMIM TFSI, which is decomposed close to room temperature in a $\mathrm{Pt}$ pan (see Figure S6 ESI $\dagger$ ). The latter is measured in an alumina crucible. The corresponding derivatives used to define $\mathrm{T}_{\text {start }}$ are plotted in Figure S5 ESI $\uparrow$. The red arrow indicates the regions where SANS and DLS are performed.

the two systems, with either $\mathrm{SBMIM}^{ \pm} \mathrm{TFSI}^{-}$or $\mathrm{SBMIM}^{ \pm}$ $\mathrm{TfO}^{-}$, differ (SANS/SAXS, DLS, TEM), this indicates that the $\mathrm{TfO}^{-}$ions localise at least in parts close to the solidliquid interface (shown in Figure 8b).

At the maximum surface charge density $\sigma$, the ratio $\kappa_{i o n} \approx 0.3$ in the case of introduced $\mathrm{TfO}^{-}$anions and $\kappa_{\text {ion }} \approx 0.6$ in the case of TFSI $^{-}$anions (with the values of ref[21, 25]). The latter being closer to 0.5 , this could explain the better colloidal stability seen in the experiments for this system, leading to 3-4 more ion layers.[22, 21] Considering the size of the ions, this matches well with the value obtained from the repulsive range which corresponds to a perturbated thickness aroung 3-5 nm around the NPs compared with the bulk structure of the IL. The lower colloidal stability of nanoparticle dispersions with $\mathrm{SBMIM}^{ \pm} \mathrm{TfO}^{-}$interfacial species could also be explained by a disturbance of the $\mathrm{SBMIM}^{ \pm}$monolayer. Both $\mathrm{SBMIM}^{ \pm}$and $\mathrm{TfO}^{-}$contain a sulfonate group having an affinity for oxide surfaces. [29, 44] A localisation of some $\mathrm{TfO}^{-}$ions would therefore disturb the monolayer of SBMIM $^{ \pm}$zwitterions due to their size difference and hence the multilayer structure formed behind, explaining the difference observed in the colloidal stability.
Looking now at lower surface charge densities $\sigma$ on sam-
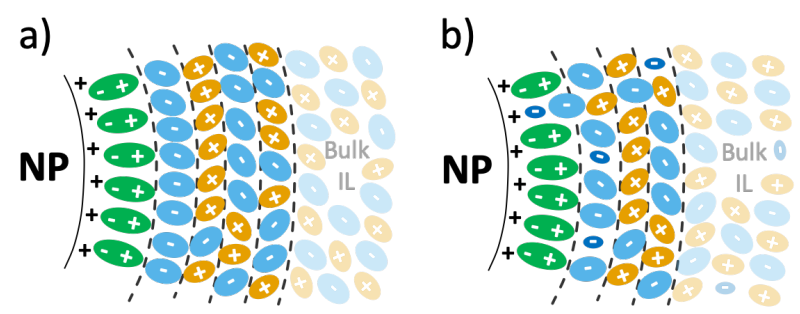

Figure 8: Schemes of the nanoparticle (NP) ionic liquid (IL) interface for an imaginable case for a) $\mathrm{SBMIM}^{ \pm} \mathrm{TFSI}^{-}$interfacial species and b) $\mathrm{SBMIM}^{ \pm} \mathrm{TfO}^{-}$interfacial species in the case of the maximal surface charge density $\sigma \approx 30 \mu \mathrm{C} / \mathrm{cm}^{2}$ depicted by several "+" on the nanoparticle surface. The ions are represented as ellipsoids, the zwitterion $\mathrm{SBMIM}^{ \pm}$in green, the cation $\mathrm{EMIM}^{+}$in yellow, and the anions $\mathrm{TFSI}^{-}$and $\mathrm{TfO}^{-}$in light and dark blue, respectively. Here $\kappa_{S B M I M \pm}=1$ $\sigma / \Theta_{S B M I M^{ \pm}}^{\max } \mid \approx 1$ leading to a layer (or more) of $\mathrm{SBMIM}^{ \pm}$ zwitterions acting as a steric component not changing the absolute charge. After the $\mathrm{SBMIM}^{ \pm}$zwitterion layer (possibly disturbed by $\mathrm{TfO}^{-}$couterions), a multilayer of the ionic liquid ions $\mathrm{EMIM}^{+}$and $\mathrm{TFSI}^{-}$(and possibly $\mathrm{TfO}^{-}$or $\mathrm{SBMIM}^{ \pm}$ions) is shown which is obtained due to overscreening of the surface charge at a ratio of $\kappa_{\text {ion }}=\left|\sigma / \Theta_{\text {ion }}^{\max }\right| \approx 0.5$. After some layers (around 3-4) the bulk ionic liquid structure is recovered, shown here with transparent ions (for more details see text).

ples at $1 \mathrm{vol} \%$ of NPs, the apparent $\mathrm{d}_{H}$ increases while the charge decreases until unstable systems are obtained. This is consistent with the decrease of the ratio $\kappa_{\text {ion }}=\left|\sigma / \Theta_{\text {ion }}^{\max }\right|$ far below 0.5 , thus a decrease of the number of organised layers. However, there is a difference between $\mathrm{SBMIM}^{ \pm} \mathrm{TfO}^{-}$ and SBMIM $^{ \pm}$TFSI $^{-}$interfacial species whatever the surface charge density $\sigma$. For lower $\sigma$, the behaviour is reversed compared to the one at the maximal charge as the NPs aggregate in smaller clusters with $\mathrm{SBMIM}^{ \pm} \mathrm{TfO}^{-}$than with SBMIM $^{ \pm} \mathrm{TFSI}^{-}$. This result also indicates that $\mathrm{TfO}^{-}$anions are close to the solid-ionic liquid interface, maybe even in the first layer of the $\mathrm{SBMIM}^{ \pm}$, modifying the organisation, producing dispersions of small aggregates instead of individual nanoparticles.

Let us now focus on thermal stability, starting with the definition of the used criteria. Many studies use TGA, and provide $\mathrm{T}_{\text {onset }}$, sometimes $\mathrm{T}_{\text {start }}$, both corresponding to shortterm behaviour under gas flow. On the long term, several criteria have been defined, one example being $1 \%$ weight loss in 10 hours $\mathrm{T}_{0.01 / 10 h}$ [8] and another being the maximum operation temperature for an annual decomposition of $1 \% \mathrm{~T}_{\text {max }, 0.01 / \text { year }}[49,20]$, a value extrapolated from isothermal measurements on much shorter duration than 1 year. All these values are available here only for EMIM TFSI (see Table 1) and show that $\mathrm{T}_{\text {start }}$ and $\mathrm{T}_{\text {onset }}$ are much larger than $\mathrm{T}_{0.01 / 10 h}$ or $\mathrm{T}_{\text {max }, 0.01 / \text { year }}$. Nevertheless, it is important to point out that evaporation can occur and is usually not taken into account (except for $\mathrm{T}_{\text {max }, 0.01 / \text { year }}$ in table 1), so that the loss of mass measured is a mixture of evaporation and degradation. Let us consider the case of EMIM TFSI. At $200^{\circ} \mathrm{C}$, 
its vapour pressure is $0.02 \mathrm{~Pa}$ (from ref [20]), however a high amount of gas passes in TGA. In 10 hour isothermal measurement, $24 \mathrm{~L}$ of gas pass on 13 micro litres (around $20 \mathrm{mg}$ ) of sample, thus $8 \times 10^{5} \mathrm{~m}_{\text {gas }}^{3} m_{\text {ionicliquid }}^{-3} h^{-1}$. If the gas saturated immediately (which is far from being the case) this would lead to complete evaporation of EMIM TFSI in around 1 minute which explains why evaporation can be observed in some ionic liquids if the carrier gas absorbs at least a bit. Note that for EMIM TFO, the vapour pressure is 0.002 $\mathrm{Pa}, 10$ times lower.

It means that the degradation is usually overestimated from TGA measurements. Therefore at our maximal temperature of analysis for the ferrofluids here in SANS $\left(200^{\circ} \mathrm{C}\right)$, EMIM TFSI can be considered as long-term stable, with no degradation and only evaporation. Another difficulty comes from the additives present in the ferrofluid, not only constituted of iron oxide and EMIM TFSI. SBMIM ${ }^{ \pm}$and HSBMIM are present as well as $\mathrm{TfO}^{-}$anions in one of the samples. Such mixtures have never been studied and the only available data concern pure ionic liquids. HSBMIM TFSI was not studied, however some ionic liquids with other anions have been tested like HSBMIM $\mathrm{HSO}_{4}$ [16] and HSPMIM TfO [1], the latter cation having one carbon separation less between the imidazolium and sulfonate functionalities. The average of their $\mathrm{T}_{\text {onset }}$ values compared to the experimental result here for HSBMIM TFSI are similar. In ionic liquids, the possible mechanisms of degradation have been explored. Chen et al.[10] analysed the temperature stability by TGA-MS of EMIM TFSI and found in their experiments that above $350^{\circ} \mathrm{C}$ first TFSI decomposes to more nucleophilic groups, such as $\mathrm{F}, \mathrm{CF}_{3}$ and $\mathrm{NH}_{2}$ that mainly attack the ethyl group of the cation EMIM by elimination and nucleophilic substitution mechanisms. The same is observed for BMIM TfO that also decomposes to methylimidazolium (MIM) and $\mathrm{HCF}_{3}$. [41] Ethyl imidazols are the first species that are detected followed by methyl imidazoles. $\mathrm{SO}_{2}$ is also detected for TFSI and as well for TfO based ionic liquids. Nakurte et al.[38] analysed the decomposition mechanisms of different zwitterions dissolved in water, one being close to the zwitterion $\mathrm{SBMIM}^{ \pm}$analysed here but with a propyl instead of a butyl side chain. ESI-MS for the compound shows protonation as the most probable. As observed in a lot of other studies for ionic liquids $[36,8,10]$ it is the longer side chain of the imidazolium ring that is cleaved in the highest amount (here 60\%) generating propane sulfonate and methyl-imidazolium. The second most probable mechanism is the elimination of sulfurous acid leaving methylpropylene-imidazolium (10\%).

From literature, it appears that the thermal stability of ionic liquids is governed by nucleophilic anion attack of the cation. Thermal stability is therefore higher for lower nucleophilicity (i.e. higher Lewis basicity) [36]. As Lewis bases (for the anion) lower the degradation temperature, it is assumed that Lewis acids (for the cation) do as well. Hence, the (H)SBMIM could be causing the formation of neutral molecules that evaporate after.

From the measurements performed here on the components of the ferrofluids and on the ferrofluids, looking at $\mathrm{T}_{\text {start }}$ and $\mathrm{T}_{\text {onset }}$, the colloidal dispersions appear less stable than pure EMIM TFSI (T shifted by $20-40^{\circ} \mathrm{C}$ ) which seems to originate from HSBMIM. However the stability is kept at $200^{\circ} \mathrm{C}$ on the scale of hours from the SANS, SAXS, DLS experiments and TGA shows limits for short-term temperature stability of around 280 to $300^{\circ} \mathrm{C}$. Moreover the long TGA measurements on $15 \mathrm{~h}$ show a good stability of the colloidal dispersions at $200^{\circ} \mathrm{C}$.

\section{Conclusions and outlook}

This work questions the design and analysis of charged (magnetic)iron oxide nanoparticles in EMIM-TFSI, a wellknown ionic liquid, fit for operating at high temperatures. Stable dispersions can be obtained up to $473 \mathrm{~K}\left(200^{\circ} \mathrm{C}\right)$, thanks to the tuning of the nanoparticle/ionic liquid interface. The key parameters for this stability are (i) the surface charge of the nanoparticles (ii) the nature of the counterions and/or interfacial species, (iii) their concentration and (iv) the particle volume fraction. The samples are analyzed on several scales by associating transmission electron microscopy (TEM), dynamic light scattering (DLS), small angle scattering of X-rays (SAXS) or neutrons (SANS).

Colloidal stability can only be achieved here in the presence of zwitterions $\mathrm{SBMIM}^{ \pm}$, species which thus locate at the solid-liquid interface, and at elevated nanoparticle surface charge, associated to either $\mathrm{TFSI}^{-}$or $\mathrm{TfO}^{-}$species, introduced by either HTFSI or HTfO.

With SBMIM $^{ \pm}$TFSI $^{-}$interfacial species the nanoparticles (NPs) are well dispersed with repulsive interparticle interactions. With $\mathrm{SBMIM}^{ \pm} \mathrm{TfO}^{-}$interfacial species, the interparticle interaction is slightly attractive and some small loose aggregates are present. Despite their different nanostructure, both systems are stable colloidal dispersions at $\Phi_{\mathrm{NP}}=1 \mathrm{vol} \%$ according to all the techniques SANS, SAXS and DLS used here. This is true also in the long term at least for one year. In addition, their colloidal stability is kept up to $473 \mathrm{~K}$. It is also the case at higher nanoparticle concentrations up to 12 vol\% for the well dispersed NPs with repulsive interparticle interaction associated with $\mathrm{SBMIM}^{ \pm} \mathrm{TFSI}^{-}$interfacial species. Hence, these long term stable ferrofluids are suited for the use in those applications which are requiring a broad range of operating temperatures.

This study demonstrates the ability to stabilize nanoparticles in an ionic liquid. The developed method can be extended to other ionic liquids as well as to other nanoparticles, based on different oxides or materials, chosen for their respective properties in order to design new task specific dispersions, in the future.

\section{Declaration of competing interest}

There are no conflicts of interest to declare. 


\section{Authors contributions}

JR did most experiments and wrote the first draft in the frame of his PhD, ED and VP supervised JR, added experiments and revised the text, JR,MS,TF,FC,ED,GM,RP,VP performed the scattering experiments. All the authors contributed to discussions and improvement of the text.

\section{Acknowledgements}

We thank Amandine Anfry for DLS measurements, Aude Michel for FAAS measurements, Arnaud Hélary for the design of the coupled DLS-SANS measurement setup at LLB (Laboratoire Léon Brillouin), and Sandra Casale for the TEM images taken at the IMPC (Institut des Matériaux de ParisCentre). We acknowledge the Laboratoire Léon Brillouin (CEA-Saclay, France) for neutron beamtime at the PAXY spetrometer. This work has been supported by the European Union Horizon 2020 research and innovation program under the grant number 731976 (MAGENTA), by the Brazilian agency $\mathrm{CNPq}$ and the bilateral program CAPES-COFECUB $\mathrm{Ph} 950 / 20$ and by the laboratories own resources.

\section{Supplementary data}

Electronic Supplementary Information (ESI) available.

\section{References}

[1] Amarasekara, A.S., Owereh, O.S., 2011. Thermal properties of sulfonic acid group functionalized Brönsted acidic ionic liquids. J Therm Anal Calorim 103, 1027-1030. URL: http://link. springer.com/10. 1007/s10973-010-1101-5, doi:10.1007/s10973-010-1101-5.

[2] Armand, M., Endres, F., MacFarlane, D.R., Ohno, H., Scrosati, B., 2011. Ionic-liquid materials for the electrochemical challenges of the future, in: Materials For Sustainable Energy: A Collection of Peer-Reviewed Research and Review Articles from Nature Publishing Group. World Scientific, pp. 129-137.

[3] Awad, W.H., Gilman, J.W., Nyden, M., Harris, R.H., Sutto, T.E., Callahan, J., Trulove, P.C., DeLong, H.C., Fox, D.M., 2004. Thermal degradation studies of alkyl-imidazolium salts and their application in nanocomposites. Thermochimica Acta 409, 3-11. URL: https: //linkinghub.elsevier.com/retrieve/pii/s0040603103003344, doi:10. 1016/S0040-6031(03)00334-4.

[4] Barker, J.A., Henderson, D., 1967. Perturbation Theory and Equation of State for Fluids. II. A Successful Theory of Liquids. The Journal of Chemical Physics 47, 4714-4721. URL: http://aip.scitation.org/ doi/10.1063/1.1701689, doi:10.1063/1.1701689.

[5] Bhattacharya, K., Sarkar, M., Salez, T.J., Nakamae, S., Demouchy, G., Cousin, F., Dubois, E., Michot, L., Perzynski, R., Peyre, V., 2020. Structural, Thermodiffusive and Thermoelectric Properties of Maghemite Nanoparticles Dispersed in Ethylammonium Nitrate. ChemEngineering 4, 5. URL: https: //www. mdpi . com/2305-7084/4/1/5, doi:10.3390/chemengineering4010005. number: 1 Publisher: Multidisciplinary Digital Publishing Institute.

[6] Bonetti, M., Nakamae, S., Huang, B.T., Salez, T.J., Wiertel-Gasquet, C., Roger, M., 2015. Thermoelectric energy recovery at ionicliquid/electrode interface. The Journal of Chemical Physics 142, 244708. URL: http://dx.doi.org/10.1063/1.4923199, doi:10.1063/1. 4923199, arXiv: http://dx.doi.org/10.1063/1.4923199.

[7] Cabreira Gomes, R., Ferreira da Silva, A., Kouyaté, M., Demouchy, G., Mériguet, G., Aquino, R., Dubois, E., Nakamae, S., Roger, M.,
Depeyrot, J., Perzynski, R., 2018. Thermodiffusion of repulsive charged nanoparticles - the interplay between single-particle and thermoelectric contributions. Phys. Chem. Chem. Phys. 20, 1640216413. URL: http://xlink.rsc.org/?DOI=C8CP02558D, doi:10.1039/ C8CP02558D.

[8] Cao, Y., Mu, T., 2014. Comprehensive Investigation on the Thermal Stability of 66 Ionic Liquids by Thermogravimetric Analysis. Ind. Eng. Chem. Res. 53, 8651-8664. URL: https://pubs.acs.org/doi/ 10.1021/ie5009597, doi:10.1021/ie5009597.

[9] Carnahan, N.F., Starling, K.E., 1970. Thermodynamic Properties of a Rigid-Sphere Fluid. The Journal of Chemical Physics 53, 600-603. URL: http://aip.scitation.org/doi/10.1063/1.1674033, doi:10.1063/ 1.1674033.

[10] Chen, Y., Cao, Y., Shi, Y., Xue, Z., Mu, T., 2012. Quantitative Research on the Vaporization and Decomposition of [EMIM][Tf 2 N] by Thermogravimetric Analysis-Mass Spectrometry. Ind. Eng. Chem. Res. 51, 7418-7427. URL: https://pubs.acs.org/doi/10. 1021/ie300247v, doi:10.1021/ie300247v.

[11] Dupont, M., MacFarlane, D., Pringle, J., 2017. Thermoelectrochemical cells for waste heat harvesting - progress and perspectives. Chem. Commun. 53, 6288-6302.

[12] Elbourne, A., McDonald, S., Voïchovsky, K., Endres, F., Warr, G.G., Atkin, R., 2015. Nanostructure of the Ionic Liquid-Graphite Stern Layer. ACS Nano 9, 7608-7620. URL: http://pubs. acs.org/doi/10. 1021/acsnano.5b02921, doi:10.1021/acsnano. 5b02921.

[13] Filomeno, C.L., Kouyaté, M., Peyre, V., Demouchy, G., Campos, A.F.C., Perzynski, R., Tourinho, F.A., Dubois, E., 2017. Tuning the Solid/Liquid Interface in Ionic Colloidal Dispersions: Influence on Their Structure and Thermodiffusive Properties. The Journal of Physical Chemistry C 121, 5539-5550. URL: http://pubs.acs.org/ doi/10.1021/acs. jpcc. 6b10280, doi:10.1021/acs. jpcc. 6b10280.

[14] França, J.M.P., Lourenço, M.J.V., Murshed, S.M.S., Pádua, A.A.H., Nieto de Castro, C.A., 2018. Thermal Conductivity of Ionic Liquids and IoNanofluids and Their Feasibility as Heat Transfer Fluids. Industrial \& Engineering Chemistry Research 57, 6516-6529. URL: https: //pubs. acs.org/doi/10.1021/acs. iecr. 7b04770, doi:10.1021/acs. iecr. $7 \mathrm{~b} 04770$.

[15] Fredlake, C.P., Crosthwaite, J.M., Hert, D.G., Aki, S.N.V.K., Brennecke, J.F., 2004. Thermophysical Properties of Imidazolium-Based Ionic Liquids. J. Chem. Eng. Data 49, 954-964. URL: https: //pubs.acs.org/doi/10.1021/je034261a, doi:10.1021/je034261a.

[16] Gui, J., Ban, H., Cong, X., Zhang, X., Hu, Z., Sun, Z., 2005. Selective alkylation of phenol with tert-butyl alcohol catalyzed by Brönsted acidic imidazolium salts. Journal of Molecular Catalysis A: Chemical 225, 27-31. URL: https://1inkinghub.elsevier.com/retrieve/ pii/S1381116904006077, doi:10.1016/j.molcata.2004.08.026.

[17] Guibert, C., Dupuis, V., Fresnais, J., Peyre, V., 2015. Controlling nanoparticles dispersion in ionic liquids by tuning the $\mathrm{pH}$. Journal of Colloid and Interface Science 454, 105-111. URL: https: //linkinghub.elsevier.com/retrieve/pii/S0021979715004361, doi:10. 1016/j.jcis. 2015.04.059.

[18] Hayes, R., Borisenko, N., Corr, B., Webber, G.B., Endres, F., Atkin, R., 2012. Effect of dissolved $\mathrm{LiCl}$ on the ionic liquid-Au(111) electrical double layer structure. Chemical Communications 48, 10246. URL: http://xlink.rsc.org/?DOI=c2cc35737b, doi:10.1039/ c2cc35737b.

[19] He, Z., Alexandridis, P., 2017. Ionic liquid and nanoparticle hybrid systems: Emerging applications. Advances in Colloid and Interface Science 244, 54-70. URL: https: //linkinghub. elsevier. com/ retrieve/pii/S0001868616302238, doi:10.1016/j.cis.2016.08.004.

[20] Heym, F., Etzold, B.J.M., Kern, C., Jess, A., 2011. Analysis of evaporation and thermal decomposition of ionic liquids by thermogravimetrical analysis at ambient pressure and high vacuum. Green Chem. 13, 1453. URL: http://xlink.rsc.org/?DOI=c0gc00876a, doi:10.1039/ c0gc00876a.

[21] Ivanistsev, V., Fedorov, M.V., 2014. Interfaces between Charged Surfaces and Ionic Liquids: Insights from Molecular Simulations. Interface magazine 23, 65-69. URL: https://iopscience.iop.org/ 
article/10.1149/2.F08141if, doi:10.1149/2.F08141if.

[22] Ivaništšev, V., O'Connor, S., Fedorov, M., 2014. Poly(a)morphic portrait of the electrical double layer in ionic liquids. Electrochemistry Communications 48, 61-64. URL: https: //linkinghub. elsevier.com/ retrieve/pii/S1388248114002690, doi:10.1016/j.elecom. 2014.08.014.

[23] Jolivet, J.P., Tronc, E., 1988. Interfacial electron transfer in colloidal spinel iron oxide. Conversion of $\mathrm{Fe} 3 \mathrm{o} 4-\mathrm{Fe} 2 \mathrm{o} 3$ in aqueous medium. Journal of Colloid and Interface Science 125, 688-701. URL: http://linkinghub.elsevier.com/retrieve/pii/ 0021979788900367, doi:10.1016/0021-9797(88)90036-7.

[24] Kamysbayev, V., Srivastava, V., Ludwig, N.B., Borkiewicz, O.J., Zhang, H., Ilavsky, J., Lee, B., Chapman, K.W., Vaikuntanathan, S., Talapin, D.V., 2019. Nanocrystals in Molten Salts and Ionic Liquids: Experimental Observation of Ionic Correlations Extending beyond the Debye Length. ACS Nano 13, 5760-5770. URL: http://pubs. acs.org/doi/10.1021/acsnano. 9b01292, doi:10.1021/acsnano. 9 b01292.

[25] Kislenko, S.A., Moroz, Y.O., Karu, K., Ivaništšev, V.B., Fedorov, M.V., 2018. Calculating the Maximum Density of the Surface Packing of Ions in Ionic Liquids. Russian Journal of Physical Chemistry A 92, 999-1005. URL: http://link.springer.com/10.1134/ s0036024418050187, doi:10.1134/S0036024418050187.

[26] Kouyaté, M., Filomeno, C.L., Demouchy, G., Mériguet, G., Nakamae, S., Peyre, V., Roger, M., Cēbers, A., Depeyrot, J., Dubois, E., Perzynski, R., 2019. Thermodiffusion of citrate-coated $\gamma$-Fe ${ }_{2} \mathrm{O}_{3}$ nanoparticles in aqueous dispersions with tuned counter-ions anisotropy of the Soret coefficient under a magnetic field. Physical Chemistry Chemical Physics 21, 1895-1903. doi:10.1039/C8CP06858E.

[27] Laux, E., Uhl, S., Jeandupeux, L., López, P.P., Sanglard, P., Vanoli, E., Marti, R., Keppner, H., 2018. Thermoelectric generators based on ionic liquids. Journal of Electronic Materials , 1-5URL: https://doi org/10.1007/s11664-018-6175-z, doi:10.1007/s11664-018-6175-z.

[28] Lopes Filomeno, C., Kouyaté, M., Cousin, F., Demouchy, G., Dubois, E., Michot, L., Mériguet, G., Perzynski, R., Peyre, V., Sirieix-Plénet, J., Tourinho, F., 2017. Ionic magnetic fluids in polar solvents with tuned counter-ions. Journal of Magnetism and Magnetic Materials 431, 2-7. URL: https://linkinghub.elsevier.com/retrieve/pii/ S0304885316315633, doi:10.1016/j.jmmm. 2016.07.050.

[29] Lu, X., Deng, S., Wang, B., Huang, J., Wang, Y., Yu, G., 2016. Adsorption behavior and mechanism of perfluorooctane sulfonate on nanosized inorganic oxides. Journal of Colloid and Interface Science 474, 199-205. URL: https://linkinghub.elsevier.com/retrieve/pii/ S0021979716302521, doi:10.1016/j.jcis.2016.04.032.

[30] Lucas, I.T., Durand-Vidal, S., Dubois, E., Chevalet, J., Turq, P., 2007. Surface Charge Density of Maghemite Nanoparticles: Role of Electrostatics in the Proton Exchange. J. Phys. Chem. C 111, 18568-18576. URL: https://pubs.acs.org/doi/10.1021/jp0743119, doi:10.1021/jp0743119.

[31] MacFarlane, D.R., Tachikawa, N., Forsyth, M., Pringle, J.M., Howlett, P.C., Elliott, G.D., Davis, J.H., Watanabe, M., Simon, P., Angell, C.A., 2014. Energy applications of ionic liquids. Energy Environ. Sci. 7, 232-250. URL: http://xlink. rsc.org/?DOI=C3EE42099J, doi:10.1039/C3EE42099J.

[32] Mamusa, M., Sirieix-Plénet, J., Cousin, F., Perzynski, R., Dubois, E., Peyre, V., 2014a. Microstructure of colloidal dispersions in the ionic liquid ethylammonium nitrate: influence of the nature of the nanoparticles' counterion. Journal of Physics: Condensed Matter 26, 284113. URL: http://stacks.iop.org/0953-8984/26/i=28/a= 284113?key=crossref . $4 f 747615 a 635 a 1 f 7 f 3 b 19376218 d 5 f 56$, doi:10.1088/ 0953-8984/26/28/284113.

[33] Mamusa, M., Sirieix-Plénet, J., Perzynski, R., Cousin, F., Dubois, E., Peyre, V., 2015. Concentrated assemblies of magnetic nanoparticles in ionic liquids. Faraday Discussions 181, 193-209. URL: http: //xlink.rsc.org/?DOI=C5FD00019J, doi:10.1039/C5FD00019J.

[34] Mamusa, M., Siriex-Plénet, J., Cousin, F., Dubois, E., Peyre, V., 2014b. Tuning the colloidal stability in ionic liquids by controlling the nanoparticles/liquid interface. Soft Matter 10, 1097. URL: http://xlink. rsc.org/?DOI=c3sm52733f, doi:10.1039/c3sm52733f.

[35] Massart, R., 1981. Preparation of aqueous magnetic liquids in alkaline and acidic media. IEEE transactions on magnetics 17, 1247-1248.

[36] Maton, C., Vos, N.D., V. Stevens, C., 2013. Ionic liquid thermal stabilities: decomposition mechanisms and analysis tools. Chemical Society Reviews 42, 5963-5977. URL: https: //pubs.rsc.org/en/ content/articlelanding/2013/cs/c3cs60071h, doi:10.1039/c3cs60071H.

[37] Mestrom, L., Lenders, J.J.M., de Groot, R., Hooghoudt, T., Sommerdijk, N.A.J.M., Artigas, M.V., 2015. Stable ferrofluids of magnetite nanoparticles in hydrophobic ionic liquids. Nanotechnology 26, 285602. URL: http://stacks. iop.org/0957-4484/26/i=28/a= 285602?key=crossref. 39900d59c9edb1754ac24b6ebb3690ec, doi:10. 1088/ $0957-4484 / 26 / 28 / 285602$.

[38] Nakurte, I., Mekss, P., Klavins, K., Zicmanis, A., Vavilina, G., Dubrovina, S., 2009. Collision-Induced Dissociation of ImidazoliumBased Zwitterionic Liquids. Eur J Mass Spectrom (Chichester) 15, 471-478. URL: http://journals. sagepub.com/doi/10.1255/ejms.997, doi:10.1255/ejms. 997.

[39] Ngo, H.L., LeCompte, K., Hargens, L., McEwen, A.B., 2000. Thermal properties of imidazolium ionic liquids. Thermochimica Acta 357-358, 97-102. URL: https://linkinghub.elsevier.com/retrieve/ pii/S0040603100003737, doi:10.1016/S0040-6031(00)00373-7.

[40] Nordström, J., Aguilera, L., Matic, A., 2012. Effect of Lithium Salt on the Stability of Dispersions of Fumed Silica in the Ionic Liquid BMImBF $_{4}$. Langmuir 28, 4080-4085. URL: http://pubs. acs.org/ doi/10.1021/la204555g, doi:10.1021/la204555g.

[41] Ohtani, H., Ishimura, S., Kumai, M., 2008. Thermal Decomposition Behaviors of Imidazolium-type Ionic Liquids Studied by Pyrolysis-Gas Chromatography. Anal. Sci. 24, 13351340. URL: http://joi.jlc. jst. go.jp/JST. JSTAGE/analsci/24.1335? from=CrossRef, doi:10.2116/analsci. 24.1335.

[42] Oliveira, F.C.C., Rossi, L.M., Jardim, R.F., Rubim, J.C., 2009. Magnetic Fluids Based on - $\mathrm{Fe}_{2} \mathrm{O}_{3}$ and $\mathrm{CoFe}_{2} \mathrm{O}_{4}$ Nanoparticles Dispersed in Ionic Liquids. The Journal of Physical Chemistry C 113, 8566-8572. URL: http://pubs.acs.org/doi/abs/10.1021/jp810501m, doi:10.1021/jp810501m.

[43] Paduszyński, K., Domańska, U., 2014. Viscosity of Ionic Liquids: An Extensive Database and a New Group Contribution Model Based on a Feed-Forward Artificial Neural Network. Journal of Chemical Information and Modeling 54, 1311-1324. URL: http://pubs.acs. org/doi/10.1021/ci500206u, doi:10.1021/ci500206u.

[44] Potapova, E., Jolsterå, R., Holmgren, A., Grahn, M., 2014. The Effect of Inorganic Ions on Dodecylbenzenesulfonate Adsorption onto Hematite: an ATR-FTIR Study. Journal of Surfactants and Detergents 17, 1027-1034. URL: http://doi.wiley.com/10.1007/ s11743-014-1576-3, doi:10.1007/s11743-014-1576-3.

[45] Riedl, J.C., Akhavan Kazemi, M.A., Cousin, F., Dubois, E., Fantini, S., Loïs, S., Perzynski, R., Peyre, V., 2020. Colloidal dispersions of oxide nanoparticles in ionic liquids: elucidating the key parameters. Nanoscale Advances 2, 1560-1572. URL: https://pubs.rsc.org/en/ content/articlelanding/2020/na/c9na00564a, doi:10.1039/C9NA00564A. publisher: Royal Society of Chemistry.

[46] Salez, T.J., Huang, B.T., Rietjens, M., Bonetti, M., Wiertel-Gasquet, C., Roger, M., Filomeno, C.L., Dubois, E., Perzynski, R., Nakamae, S., 2017. Can charged colloidal particles increase the thermoelectric energy conversion efficiency? Physical Chemistry Chemical Physics 19, 9409-9416. URL: http://xlink.rsc.org/?DOI=C7CP01023K, doi:10. 1039/C7CP01023K.

[47] Sarkar, M., Riedl, J.C., Demouchy, G., Gélébart, F., Mériguet, G., Peyre, V., Dubois, E., Perzynski, R., 2019. Inversion of thermodiffusive properties of ionic colloidal dispersions in water-DMSO mixtures probed by forced Rayleigh scattering. Eur. Phys. J. E 42, 72. URL: https://doi.org/10.1140/epje/i2019-11835-6, doi:10.1140/ epje/i2019-11835-6.

[48] Schwertmann, U., Cornell, R.M., 2008. Iron Oxides in the Laboratory: Preparation and Characterization. John Wiley \& Sons. GoogleBooks-ID: yfOrDwOvCYkC.

[49] Seeberger, A., Andresen, A.K., Jess, A., 2009. Prediction of longterm stability of ionic liquids at elevated temperatures by means of non-isothermal thermogravimetrical analysis. Phys. Chem. Chem. 
Phys. 11, 9375. URL: http://xlink.rsc.org/?D0I=b909624h, doi:10. 1039/b909624h.

[50] Villanueva, M., Coronas, A., García, J., Salgado, J., 2013. Thermal Stability of Ionic Liquids for Their Application as New Absorbents. Ind. Eng. Chem. Res. 52, 15718-15727. URL: https://pubs. acs.org/ doi/10.1021/ie401656e, doi:10.1021/ie401656e.

[51] Wandersman, E., Cēbers, A., Dubois, E., Mériguet, G., Robert, A., Perzynski, R., 2013. The cage elasticity and under-field structure of concentrated magnetic colloids probed by small angle X-ray scattering. Soft Matter 9, 11480. URL: http://xlink.rsc.org/?DOI= c3sm51961a, doi:10.1039/c3sm51961a.

[52] Welton, T., 1999. Room-Temperature Ionic Liquids. Solvents for Synthesis and Catalysis. Chemical Reviews 99, 2071-2084. URL: http: //pubs.acs.org/doi/abs/10.1021/cr980032t, doi:10.1021/cr980032t.

[53] Werner, S., Haumann, M., Wasserscheid, P., 2010. Ionic Liquids in Chemical Engineering, in: Prausnitz, JM and Doherty, MF and Segalman, RA (Ed.), Annual Review of Chemical and Biomolecular Engineering, Vol 1. Annual Reviews. volume 1 of Annual Review of Chemical and Biomolecular Engineering, pp. 203-230. doi:10.1146/ annurev-chembioeng-073009-100915.

[54] Zhang, H., Dasbiswas, K., Ludwig, N.B., Han, G., Lee, B., Vaikuntanathan, S., Talapin, D.V., 2017. Stable colloids in molten inorganic salts. Nature 542, 328-331. URL: http://www. nature.com/articles/ nature21041, doi:10.1038/nature21041. 


\title{
Design of long-term stable concentrated colloidal dispersions in ionic liquids up to $473 \mathrm{~K}$
}

\author{
J. C. Riedl ${ }^{1}$, M. Sarkar ${ }^{1}$, T. Fiuza ${ }^{1,2}$, F. Cousin ${ }^{3}$, J. Depeyrot ${ }^{2}$, E. Dubois ${ }^{1, *}$, G. Mériguet ${ }^{1}$, R. \\ Perzynski $^{1}$, and V. Peyre ${ }^{1, *}$
}

${ }^{1}$ Sorbonne Université, Laboratoire PHENIX, 4 place Jussieu, case 51, 75005, Paris, France

${ }^{2}$ Inst. de Fisica, Complex Fluid Group, Universidade de Brasília, Brasília, Brazil

${ }^{3}$ Laboratoire Léon Brillouin, UMR CNRS 12, CE Saclay, Gif sur Yvette, France

*emmanuelle.dubois@sorbonne-universite.fr, veronique.peyre@sorbonne-universite.fr

\section{SUPPLEMENTARY INFORMATION}

\section{S1 Materials}

The following materials were used without further purification: Sodium hydroxide (NaOH pellets, 98\%, Acros Organics); hydrochloric acid ( $\mathrm{HCl}, 37 \%$ water solution, AnalaR Normapur); iron(II) chloride $\left(\mathrm{FeCl}_{2} * 4 \mathrm{H}_{2} \mathrm{O}\right.$, AnalaR Normapur, VWR); iron(III) chloride $\left(\mathrm{FeCl}_{3} * 6 \mathrm{H}_{2} \mathrm{O}\right.$, Prolabo); iron(III) nitrate $\left(\mathrm{Fe}\left(\mathrm{NO}_{3}\right)_{3} * 9 \mathrm{H}_{2} \mathrm{O}\right.$, technical, VWR); triflic acid (HTfO, $49.2 \mathrm{wt} \%$ in water, purity 99\%, SOLVIONIC); N,N-Bis(trifluoromethanesulfonyl)imide acid (HTFSI, purity 95\%, Fluorochem); 1-(4-Sulfobutyl)-3-methylimidazolium bistriflimide (HSBMIM TFSI, purity 98\%, SOLVIONIC); 1-(4-Sulfobutyl)-3methylimidazolium triflate (HSBMIM TfO, purity 98\%, SOLVIONIC); 1-(4-Sulfobutyl)-3-methylimidazolium (SBMIM ${ }^{ \pm}$, purity $98 \%$, SOLVIONIC).

\section{S2 Colloidal stabilities at $\Phi_{\mathrm{NP}} \simeq 1 \%$}

This section presents additional results about the colloidal stability. Firstly, Figure S1 presents SAXS results for some samples presented in Figure 2 of the main text, and this SAXS supports the DLS results. Indeed, the doubt in DLS results from the uncertainty on the viscosity of the medium in the present case. An apparent hydrodynamic diameter is extracted, using the Stokes-Einstein relation:

$$
D=\frac{k_{\mathrm{B}} T}{3 \pi \eta d_{H}}
$$

with the Boltzmann constant $\mathrm{k}_{\mathrm{B}}$, the absolute temperature $\mathrm{T}$, and the solvent shear-viscosity $\eta$. In the calculation of the apparent hydrodynamic diameter $\mathrm{d}_{H}$ (apparent because the interparticle interaction is forgotten in this equation) only the viscosity of EMIM TFSI is considered. However in the studied dispersions, HTFSI is introduced first to tune the NPs' charge, then $\mathrm{SBMIM}^{ \pm}$is added to vary the ratio between $\mathrm{TFSI}^{-}$and $\mathrm{SBMIM}^{ \pm}$(two step process). Part of the $\mathrm{SBMIM}^{ \pm}$move to the NPs interface, and the other part can either stay in the IL or can catch an $\mathrm{H}^{+}$to form HSBMIM. In this latter case, the dispersing medium becomes a mixture of EMIM TFSI and HSBMIM TFSI, an ionic liquid with a very high viscosity $(\approx 1700 \mathrm{cP}$ at 25 $\left.{ }^{\circ} \mathrm{C}\right)$. It would thus change the viscosity of the dispersing medium by a factor up to 2 if we assume a linear influence on the viscosity and the maximal amount of HSBMIM TFSI in the solvent. Hence, the apparent hydrodynamic diameter plotted could be overestimated by a factor of up to 2. SAXS enables to separate the existence of aggregates from hidden viscosity effects. Figure $S 1$ shows that the $S(Q \rightarrow 0)$ values reproduce the same trend as $d_{H}$ however less pronounced. This confirms that it is mainly the nanostructure that changes, however, together with an additional influence of the viscosity with increasing SBMIM $^{ \pm}$ concentration.

Secondly, Figure S1 shows that several sample preparations with the same composition are perfectly reproducible, the SAXS curves being superimposed. This was done on samples at the maximal charge with the "one step process" adding the HSBMIM TFSI ionic liquid directly, which also improves reproducibility.

Thirdly, SAXS supports the DLS results in the long run as shown in Figure S2. The colloidal stability of both nanoparticle dispersions with $\mathrm{SBMIM}^{ \pm} \mathrm{TFSI}^{-}$species and $\mathrm{SBMIM}^{ \pm} \mathrm{TfO}^{-}$species is kept for a year (at room temperature) despite 


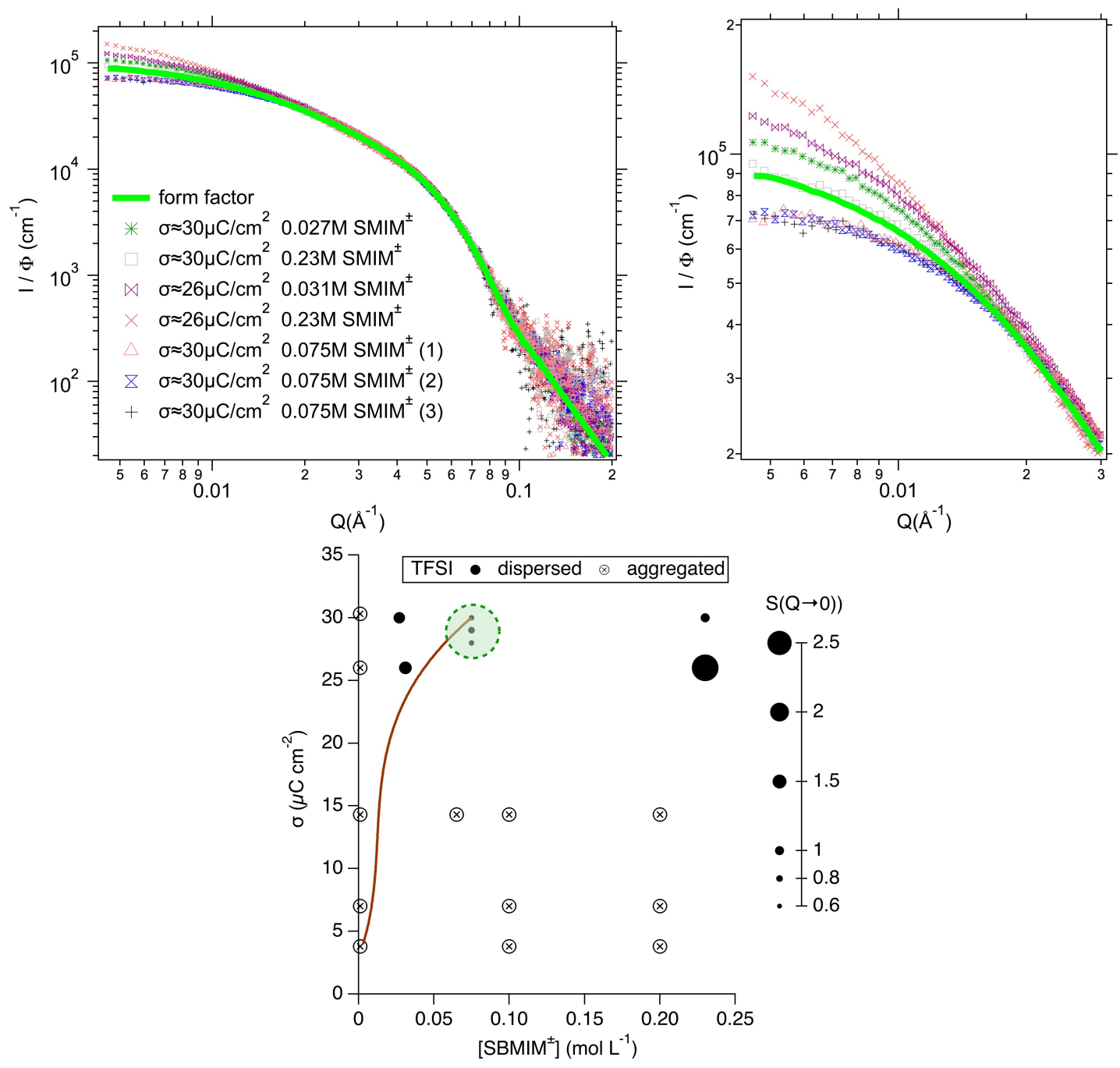

Figure S1. Top) Absolute scattered intensities (normalized by the nanoparticle volume fraction $\Phi_{\mathrm{NP}} \approx 0.8-1$ vol\%) as a function of Q obtained by SAXS for nanoparticle dispersions in EMIM TFSI with SBMIM ${ }^{ \pm}$TFSI $^{-}$interfacial species in different ratios and concentrations. The samples are prepared with the two step process except the last three, marked(1),(2), and (3). They are prepared with the one step process in the same way to test the reproducibility (see text for details). The figure on the right is a zoom of the small Q region that clearly shows the difference between the samples as well as the good reproducibility for several independent preparations; Bottom) The corresponding $\mathrm{S}(\mathrm{Q} \rightarrow 0)$ values for the samples which are not aggregated. The brown line corresponds to a ratio 1 between $\mathrm{SBMIM}^{ \pm}$and $\mathrm{TFSI}^{-}$. The green area points towards the best parameters $\sigma$ and $\left[\mathrm{SBMIM}^{ \pm}\right.$] to obtain the most repulsive samples with well dispersed NPs.

their difference in interparticle interaction. Samples left in contact with the atmosphere did not show any significant evolution either. 


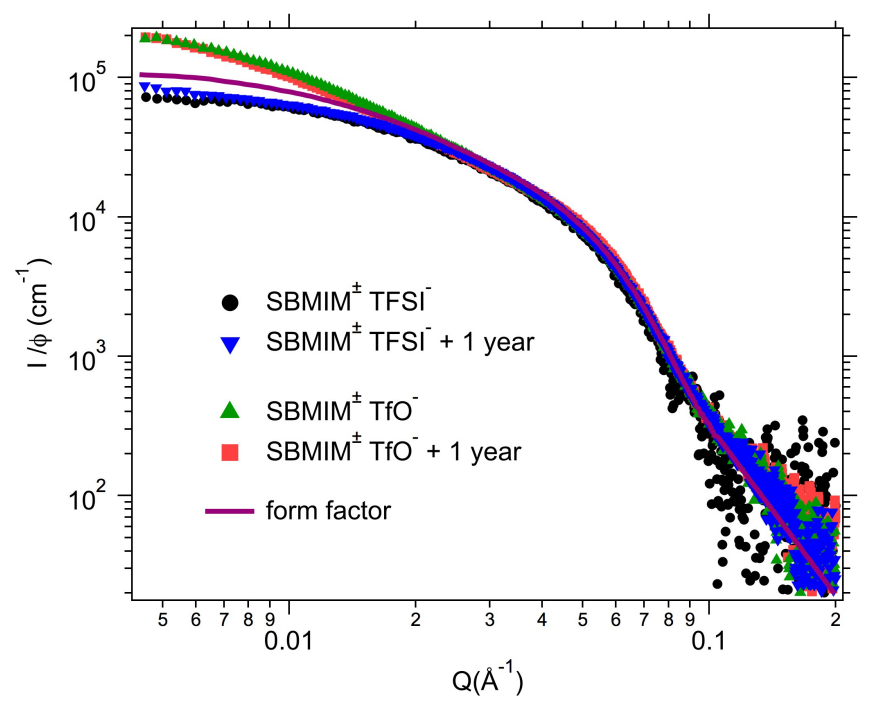

Figure S2. Absolute scattered intensities (normalized by the nanoparticle volume fraction $\Phi_{\mathrm{NP}} \approx 1$ vol\%) as a function of $\mathrm{Q}$ obtained by SAXS for dispersions of nanoparticles in EMIM TFSI with SBMIM ${ }^{+-} \mathrm{TFSI}^{-}$and $\mathrm{SBMIM}^{+/-} \mathrm{TfO}^{-}$counter-ions measured more than one year apart. During this time the sample was stored at ambient temperature and the sample with SBMIM $^{+-}$TFSI ${ }^{-}$was additionally in contact with (the moisture of) air.

\section{S3 Thermal Stabilities}

\section{S3.1 Coupled SANS and DLS measurements}

DLS and SANS measurements were coupled at temperatures up to $473 \mathrm{~K}\left(200^{\circ} \mathrm{C}\right)$. Figure $\mathrm{S} 3$ shows the setup of the experiments performed at PAXY, LLB. A furnace was fixed on a controlled rotating table to ensure a reproducible position for all samples. The oven was filled with nitrogen gas to work under inert atmosphere. A self-designed support for a DLS laser remote source (from Cordouan Vascoflex or VascoKin) was fixed on the rotating table, Figure S3 d. Figure S3 e shows the DLS remote laser source fixed on the support after turning the table by $45^{\circ}$ and with a laser protection for security. As DLS measurements are fast (around $2 \mathrm{~min}$ for ionic liquid-based colloids analysed here) the samples were analysed during temperature changes to monitor temperature variations inside the sample. After obtaining a constant temperature, i.e., a constant DLS signal, the DLS remote laser was removed. The table was turned back to the initial position and the SANS measurement was performed. These steps were repeated at $27^{\circ} \mathrm{C}, 70^{\circ} \mathrm{C}, 135^{\circ} \mathrm{C}, 200^{\circ} \mathrm{C}$ and back to $27^{\circ} \mathrm{C}$. As only classical circular quartz/spacer/quartz sandwich (see Figure S3a) could fit in this furnace and no change of the furnace was possible, adapted cells were developed, making it possible to heat liquid samples in the furnace. The cells are based on a Teflon spacer glued on quartz with a hole on the top to evacuate gases that may escape during the heating process. Figure S3b shows a filled cell and in c) the cell was placed in the oven.

Leaks occurred with concentrated samples at high temperature as mentionned in the main text. Such a leak already appeared with dilute samples $\left(\Phi_{\mathrm{NP}} \approx 1 \mathrm{vol} \%\right)$ during the tests of the cell and was solved by adapting the nature of the ring spacer (and the nature of the materials). It appeared as a mixture of wetting phenomena and bubbling/degasing at high temperature, which was not occurring in the same way depending on the nature of the materials used to build the cell. This result shows however that these wetting phenomena and bubbling/degasing grow with $\Phi_{\mathrm{NP}}$.

Three neutron runs were performed during which three configurations were used that slightly differed:

1st run) neutron wavelength $\lambda=6 \AA$, sample to detector distance $\mathrm{d}=1 \mathrm{~m} ; \lambda=6 \AA$, $\mathrm{d}=3 \mathrm{~m}$ and $\lambda=8.5 \AA$, $\mathrm{d}=5 \mathrm{~m}$

2nd run) $\lambda=5 \AA, d=1 \mathrm{~m} ; \lambda=5 \AA, d=3 \mathrm{~m}$ and $\lambda=8.5 \AA, d=5 \mathrm{~m}$

3rd run) $\lambda=4 \AA, d=1 \mathrm{~m} ; \lambda=5 \AA, d=3 \mathrm{~m}$ and $\lambda=8.5 \AA, d=5 \mathrm{~m}$

DLS enables checking that equilibrium is reached before measuring SANS. This is possible as the viscosity drastically varies with temperature which influences the correlation function. Hence, stability of the correlation function means that the viscosity and temperature are stable. DLS performed after SANS tells us whether some change occurred during the SANS measurement. In addition, agglomeration not visible by eye or large viscosity changes due to a chemical evolution also invisible by eye could be detected if curves drift to longer/shorter correlation lengths and hence time could be saved by skipping some SANS measurements. Figure $\mathrm{S} 4$ shows an example for nanoparticle dispersions with $\mathrm{SBMIM}^{ \pm} \mathrm{TFSI}^{-}$interfacial species in EMIM TFSI. The change of the correlation functions $\mathrm{G}_{1}(\mathrm{t})$ is driven by viscosity, leading to a shift of the correlation function without 

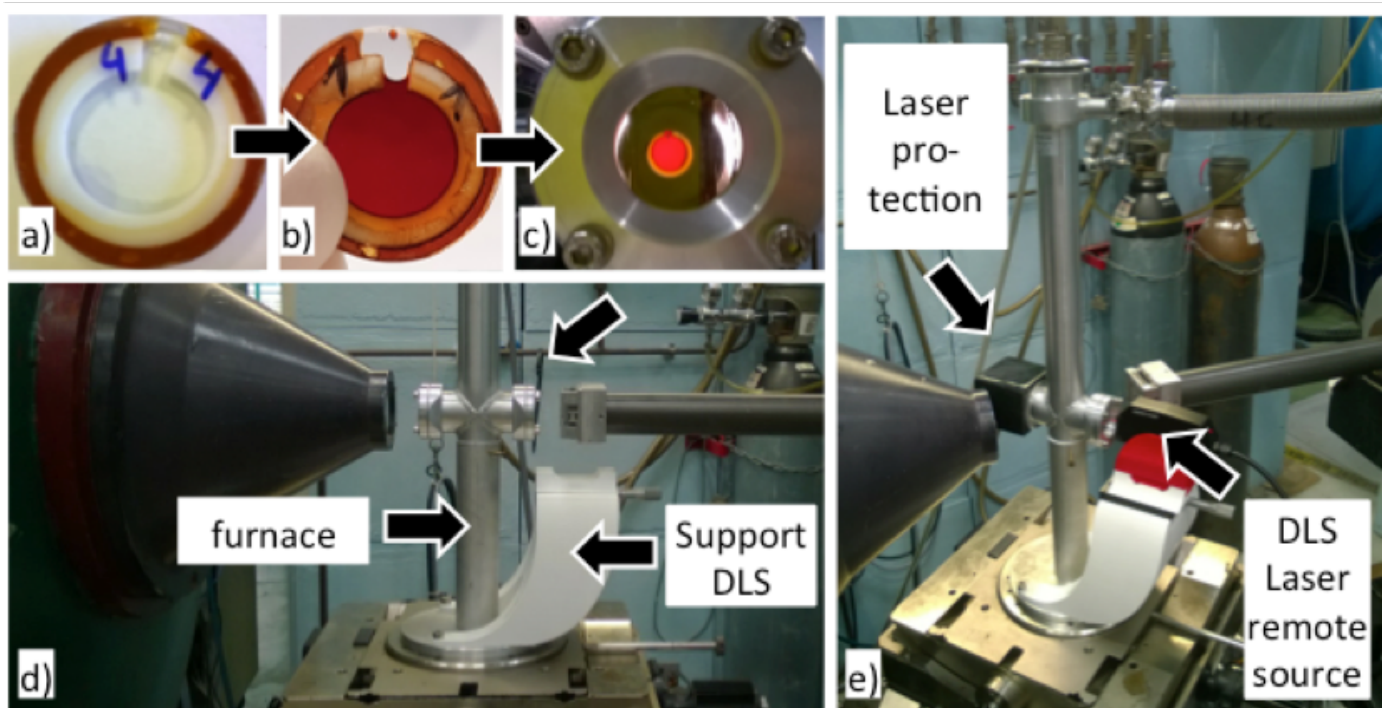

Figure S3. Scheme of the coupled SANS and DLS measurement setup at the PAXY spectrometer. a) Circular quartz/spacer/quartz sandwich with a hole on the top to evacuate gases that may form during the heating process at elevated temperatures. In b) this cell is filled with a sample and in c) it is in the furnace. d) Shows the configuration for SANS measurements and e) for DLS measurements.

distortion. Moreover, the two correlation functions before and after heating to $200^{\circ} \mathrm{C}$ superimpose (ignoring a measurement problem due to vibrations leading to oscillations in the signal).

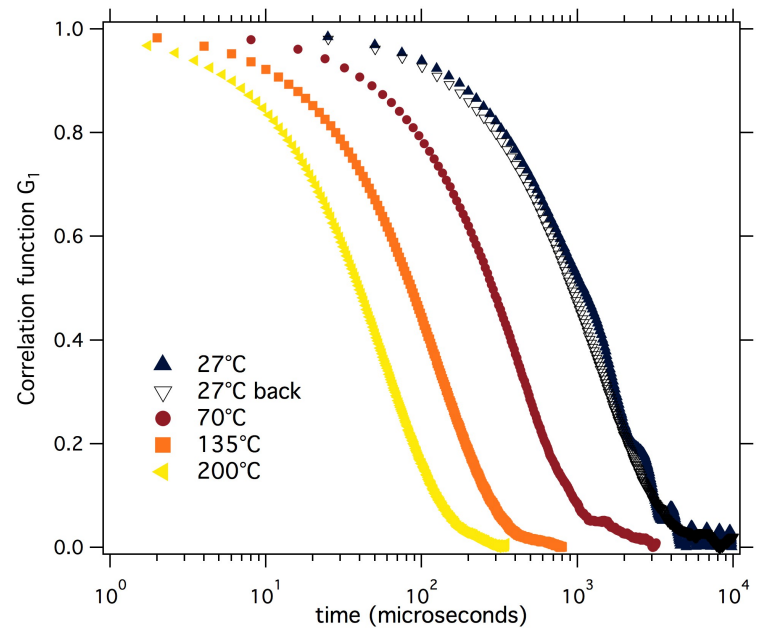

Figure S4. Dynamic light scattering (DLS) measurements for nanoparticle dispersions with $\mathrm{SBMIM}^{ \pm} \mathrm{TFSI}^{-}$interfacial species in EMIM TFSI. Note that the shift of the curves is due to a change of viscosity due to the variation of temperature.

\section{S3.2 Thermogravimetric analysis (TGA)}

Figure S5 presents the derivative mass loss corresponding to the data plotted in Figure 7 of the main text. It is used to determine $\mathrm{T}_{\text {start }}$.

As mentioned in the main text, HSMIM TFSI measured in classical Pt crucibles already decomposes at $30^{\circ} \mathrm{C}$ as can be seen in Figure S6. This can be due to a catalysis due to Pt, as this has been observed for aluminum for example in ILs, a material widely used for disposable pans ${ }^{1}$. Alumina crucibles are better however ILs anyway tend to escape from the crucibles. As these crucibles have to be set in the Pt pans due to the conception of the device, a contact between the samples and the Pt can never be completely excluded. 


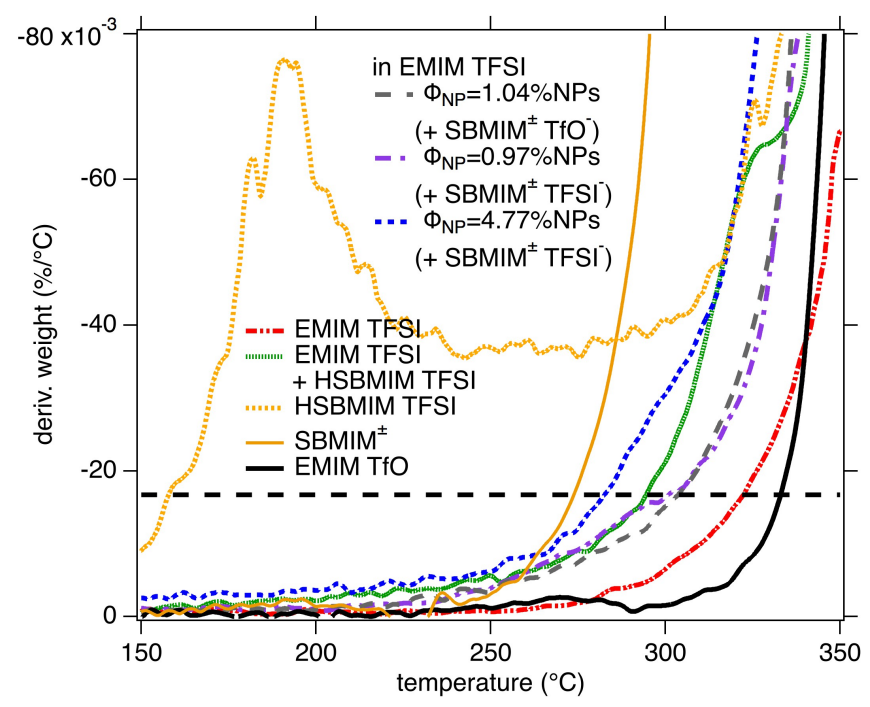

Figure S5. Ramped thermogravimetric analysis (TGA) measurements for the components of the nanoparticle dispersions in EMIM TFSI and for the resulting dispersions in EMIM TFSI. Pure EMIM TFSI and EMIM TfO are wet to mimic the worst case in the dispersions. Dry SBMIM ${ }^{ \pm}$and dry EMIM TFSI containing 2.5wt\% HSBMIM TFSI are also depicted, $2.5 \mathrm{wt} \%$ HSBMIM TFSI being the amount added while preparing the nanoparticle dispersions at $1 \mathrm{vol} \%$ nanoparticles. The final dispersions with $\Phi_{\mathrm{NP}}=1.04$ vol $\%$ with $\mathrm{SBMIM}^{ \pm} \mathrm{TfO}^{-}$interfacial species as well as $\Phi_{\mathrm{NP}}=0.97 \mathrm{vol} \%$ and a concentrated sample with $\Phi_{\mathrm{NP}}=4.77 \mathrm{vol} \%$ nanoparticles with $\mathrm{SBMIM}^{ \pm} \mathrm{TFSI}^{-}$are also plotted. $\mathrm{T}_{\text {start }}$ is here defined for a derivative mass loss of $1.67 \times 10^{-2} \% /{ }^{\circ} \mathrm{C}$ representing a weight loss of $10 \% / 1 \mathrm{~h}$ and is indicated in the graph by the horizontal black dashed line. All the measurements are performed in PT pans except HSBMIM TFSI for which an alumina crucible was used (see Figure S6 for more details).

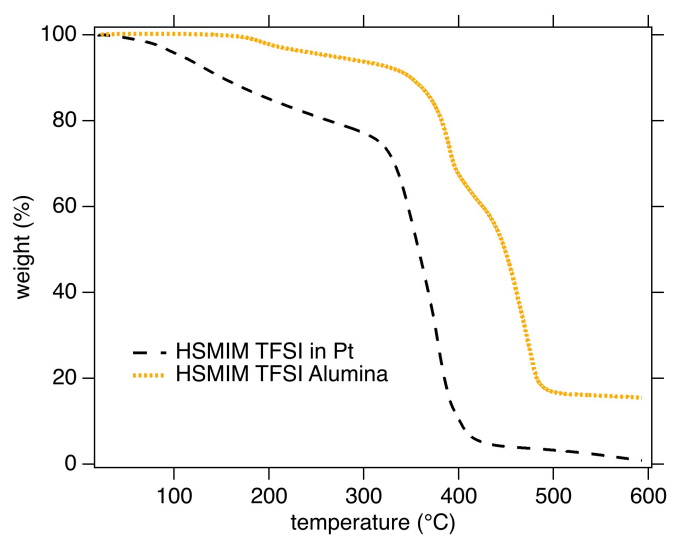

Figure S6. Ramped thermogravimetric analysis (TGA) measurements for HSBMIM TFSI in a Pt pan and HSBMIM TFSI in an alumina crucible. This latter has a cover contrary to the Pt pan, which can limit evaporation if it occurs.

Isothermal TGA measurements are shown in Figure S7. For EMIM TFO, after a first heating to $160^{\circ} \mathrm{C}$ at $20^{\circ} \mathrm{C} / \mathrm{min}$, the weight loss is measured during three hours at $160^{\circ} \mathrm{C}$, followed by three hours at $180^{\circ} \mathrm{C}$ and then ten hours at $200^{\circ} \mathrm{C}$. The weight loss is lower than $0.1 \%$, showing that it is long-term stable at these temperatures, here on 16 hours. A Pt pan was used.

For EMIM TFSI and the ferrofluids, the samples were heated at $20^{\circ} \mathrm{C} / \mathrm{min}$ to $200^{\circ} \mathrm{C}$ before measuring the weight at $200^{\circ} \mathrm{C}$ during 15h. It was done in a Pt pan and in an alumina crucible. For the pure EMIM TFSI, the container has a weak influence, as opposed to the colloidal dispersions. This is attributed to the evolution of the additive HSBMIM in Pt pans shown in Figure S6. Therefore the more exact and relevant measurements for our colloidal dispersions are the one performed in the alumina crucibles. 


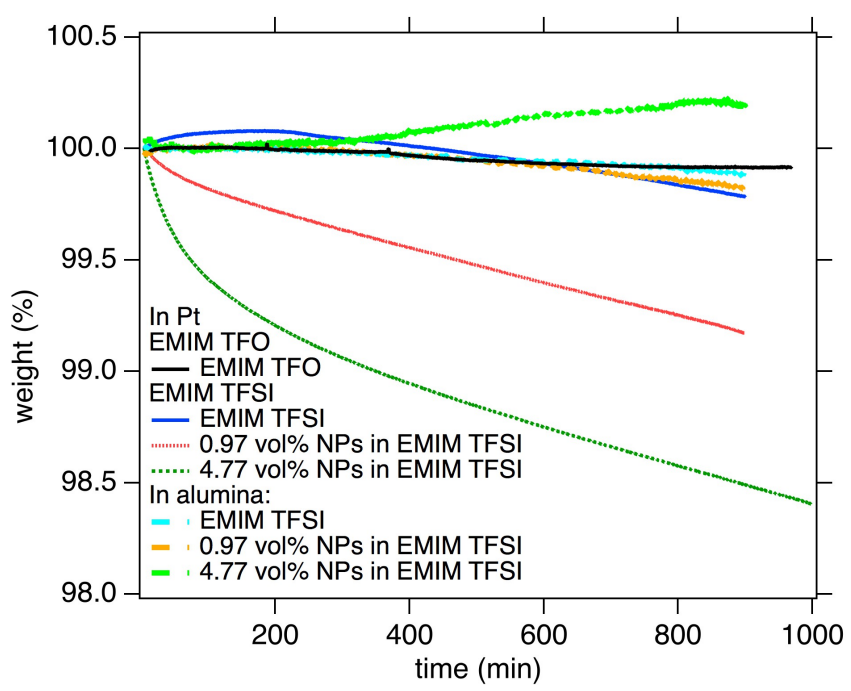

Figure S7. Isothermal thermogravimetric analysis (TGA) measurements for EMIM TfO, EMIM TFSI and the derived colloidal dispersions in two different containers. See legend and text for details.

\section{References}

1. Ngo, H. L., LeCompte, K., Hargens, L. \& McEwen, A. B. Thermal properties of imidazolium ionic liquids. Thermochimica Acta 357-358, 97-102, DOI: 10.1016/S0040-6031(00)00373-7 (2000). 